\title{
氧气参与的无张力碳-碳单键断裂反应研究进展
}

\author{
吴空宋婵崔冬梅* \\ (浙江工业大学药学院 杭州 310014)
}

\begin{abstract}
摘要 无张力碳一碳单键广泛存在于各类有机化合物中，该类型键断裂反应的研究是有机合成最重要同时也是最具有 挑战性的课题之一. 氧化断裂是无张力碳一碳单键断裂主要方式, 特别是过渡金属催化的氧化断裂反应, 近年来取得了 重大进展. 氧气作为最廉价、环保的氧化剂，已广泛用于各类有机化学反应. 根据是否需要过渡金属催化，分类综述了 近十年来氧气参与的无张力碳一碳单键断裂反应研究进展.
\end{abstract}

关键词 氧气; 无张力碳一碳单键; 断裂

\section{Advances of Unstrained Carbon-Carbon Single Bond Cleavage with Oxygen}

\author{
$\mathrm{Wu}$, Kong Song, Chan Cui, Dongmei* \\ (College of Pharmaceutical Science, Zhejiang University of Technology, Hangzhou 310014)
}

\begin{abstract}
Unstrained carbon-carbon single bonds are ubiquitous in organic compounds, the cleavage of this bond is one of the most significant and challenging subject in organic chemistry. Oxidative cleavage of unstrained carbon-carbon single bond has become a great tendency, in particular, the transition metal-catalyzed oxidative cleavage reaction, which had made significant progress in recent years. Oxygen, as the most inexpensive and environmentally friendly oxidant, has been widely used in various organic reactions. This review is an overview of recent advances of unstrained carbon-carbon single bond cleavage with oxygen according to whether transition metal catalysis is needed.
\end{abstract}

Keywords oxygen; unstrained carbon-carbon single bond ; cleavage

无张力的碳一碳单键是有机化合物中最基础、最广 泛的一类化学键, 这一类型键的断裂反应已被广泛应用 于天然产物的合成 ${ }^{[1]}$ 、新能源开发 ${ }^{[2]}$ 以及生物降解 ${ }^{[3]}$ 等领 域, 因而备受关注. 过渡金属催化的无张力碳-碳单键 氧化断裂是该类反应发展的大趋势，与此同时，非金属 催化的碳一碳单键氧化断裂反应也有了一定的进展 ${ }^{[4]}$. 碳一碳单键氧化断裂一般需要氧化剂参与, 传统的氧化 剂(如 $\mathrm{KMnO}_{4}$ 、过氧化物等)往往投料大、反应剧烈不容 易控制, 还生成大量无机废料, 给环境增加了一定的负 担. 氧气作为最廉价易得、环境友好的氧化剂, 已经广 泛应用于各种有机化学反应. 目前还没有从氧气参与该 类反应的角度对无张力碳一碳单键断裂反应进行综述, 本文根据是否使用过渡金属催化分类综述了氧气参与 该类反应的研究进展, 着重探究氧气在该类反应中所起 的作用以及碳一碳单键是如何断裂的. 通过综述总结, 希望对氧气应用于该类型反应机理有更深的理解或有
所启发.

\section{1 氧气参与过渡金属催化的无张力碳-碳单键 断裂反应}

近年来，过渡金属(如 $\mathrm{Cu} 、 \mathrm{Fe} 、 \mathrm{~V} 、 \mathrm{Mn} 、 \mathrm{Pd}$ 等)催 化的有机反应取得了重大进展，特别是过渡金属催化 碳一碳单键形成偶联反应(如 Suzuki ${ }^{[5]}$ 、 Heck ${ }^{[6]}$ 以及 Sonogashi ${ }^{[7]}$ 等反应) 的发现和发展, 为有机合成提供了 更多新的合成途径. 相比之下, 无张力碳一碳单键断裂 反应研究进展要缓慢得多，但过渡金属在该类反应中同 样发挥着极其重要的作用, 过渡金属催化也是该类反应 研究主要方向. 氧气作为最廉价、环保的试剂, 在无张 力碳-碳单键断裂反应中主要作为氧化剂将催化金属氧 化或与底物生成不稳定的过氧化物或过氧化自由基而 发挥作用.根据碳-碳单键杂化不同类型，将氧气参与的 过渡金属催化无张力碳-碳单键断裂反应分为以下三大

\footnotetext{
* Corresponding author. E-mail: cuidongmei@zjut.edu.cn

Received September 28, 2016; revised October 28, 2016; published online November 17, 2016.
} 
类进行综述: $\mathrm{C}\left(\mathrm{sp}^{3}\right)-\mathrm{C}\left(\mathrm{sp}^{3}\right)$ 单键断裂反应, $\mathrm{C}\left(\mathrm{sp}^{2}\right)(\mathrm{CO})$ $\mathrm{C}\left(\mathrm{sp}^{3}\right)$ 单键断裂反应, $\mathrm{C}\left(\mathrm{sp}^{2}\right)(\mathrm{CO})-\mathrm{C}\left(\mathrm{sp}^{2}\right)$ 和 $\mathrm{C}\left(\mathrm{sp}^{2}\right)$ $(\mathrm{CO})$ - $\mathrm{C}(\mathrm{sp})$ 单键断裂反应.

\section{$1.1 \mathrm{C}\left(\mathrm{sp}^{3}\right)-\mathrm{C}\left(\mathrm{sp}^{3}\right)$ 单键断裂反应}

由于热力学稳定性和动力学惰性, 无张力的 $\mathrm{C}\left(\mathrm{sp}^{3}\right)-\mathrm{C}\left(\mathrm{sp}^{3}\right)$ 单键的断裂比较困难, 但当碳-碳单键一 端或两端的碳上连有杂原子(如 $\mathrm{N}, \mathrm{O}, \mathrm{P}$ 等)时, 该类键在 一定程度上被活化, 促进了该类型碳一碳单键的断裂.

2009 年, Baker 和 Thorn 课题组 ${ }^{[8]}$ 首次在空气下使 用 $\mathrm{V}(\mathrm{V}) /$ 吡啶二甲酸复合物催化频哪醇 $\mathrm{C}\left(\mathrm{sp}^{3}\right)-\mathrm{C}\left(\mathrm{sp}^{3}\right)$ 单键氧化断裂, 生成两分子丙酮(Eq. 1). 该反应有望应 用于自然界中广泛存在的木质素和纤维素的氧化水解, 以解决当前的能源问题.

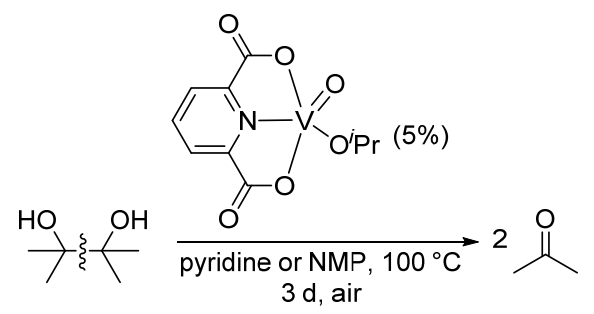

2010 年, Kirihara 课题组 ${ }^{[9]}$ 报道了 V(V)[如 VO$(\mathrm{OEt}) \mathrm{Cl}_{2}, \mathrm{VOCl}_{3}$ ]催化多取代的乙二醇类化合物发生 $\mathrm{C}\left(\mathrm{sp}^{3}\right)-\mathrm{C}\left(\mathrm{sp}^{3}\right)$ 单键氧化断裂, 高效率地生成两分子酮 的方法(Eq. 2). 该反应在氧气中室温即可反应, 条件相 对温和, 而且适用于多种不同的底物.

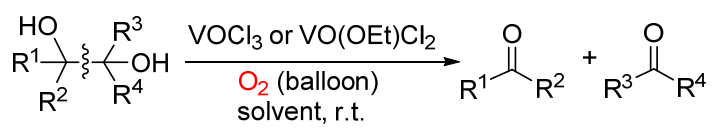

2013 年, Cavani 课题组 ${ }^{[10]}$ 报道了 $\mathrm{Ru}(\mathrm{OH})_{3}$ 作为催 化剂, 氧气作为氧化剂, 将反式 1,2-环己二醇氧化成己 二酸的方法(Scheme 1). Cavani 等认为 $\mathrm{Ru}(\mathrm{III})$ 先将反式 1,2-环已二醇催化氧化成 1,2-环已二酮, 再进一步氧化 为目标化合物. 反应中 $\mathrm{Ru}(\mathrm{OH})_{3}$ 是附着在矾石的固体催 化剂, 故可以回收再利用.

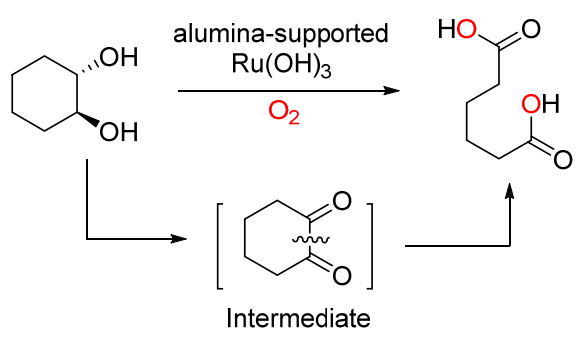

图式 $1 \mathrm{Ru}$ 催化 1,2-环己二醇生成己二酸

Scheme 1 Ru catalyzed synthesis of adipic acid from 1,2-cyclohexanediol
2012 年, Liu 课题组 ${ }^{[11]}$ 意外地发现在氧气或空气 下，一价 $\mathrm{Cu}$ 盐催化二氧六环其中一个 $\mathrm{C}\left(\mathrm{sp}^{3}\right)-\mathrm{C}\left(\mathrm{sp}^{3}\right)$ 单 键发生氧化断裂, 然后与羧酸或醛偶联分别生成 $\alpha$-酰氧 基醚或 1,2-乙二醇甲酸酯类化合物. 该反应是首次报道 的 $\mathrm{Cu}$ 催化的醚中饱和 $\mathrm{C}\left(\mathrm{sp}^{3}\right)-\mathrm{C}\left(\mathrm{sp}^{3}\right)$ 单键的断裂，通过 该方法他们合成了 42 个收率在 $43 \% \sim 99 \%$ 的目标化合 物(Eq. 3).

$$
\underset{\mathrm{Ar}(\mathrm{R})-\mathrm{CHO}}{\mathrm{Ar}(\mathrm{R})-\mathrm{COOH}}
$$

Liu 等认为该反应可能经由 Scheme 2 中的机理进 行：首先 $\mathrm{Cu}(\mathrm{I})$ 被氧气氧化生成 $\mathrm{Cu}(\mathrm{II})$ 过氧化自由基，该 自由基夺去二氧六环其中一个亚甲基上的氢原子生成 自由基中间体 $\mathbf{I}$ ，此步是反应的关键步骤. 自由基 $\mathbf{I}$ 捕获 一分子氧生成过氧化自由基 II, II 继续与氧气反应得到 自由基 III 或直接与醛、羧酸生成化合物 IV, 自由基 III 再通过电子转移和由 $\mathrm{Cu}$ 催化的碳-氢均裂反应, 最终生 成 $\mathrm{C}\left(\mathrm{sp}^{3}\right)-\mathrm{C}\left(\mathrm{sp}^{3}\right)$ 单键氧化断裂产物 $\mathbf{V}$. 氧气在该反应 中不仅作为氧化剂将 $\mathrm{Cu}(\mathrm{I})$ 氧化, 还参与过氧化自由基 的生成, 促使碳一碳单键发生断裂.

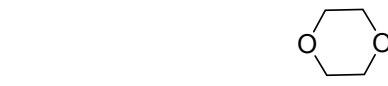<smiles>O=CCC(CO)CCl</smiles><smiles>CCCCOC(CO)COO</smiles><smiles>[R]C(=O)OC1COCCO1</smiles><smiles>[R]OC(=O)OCC(=O)OCC</smiles><smiles>C1COCCO1</smiles><smiles>[AlH2]</smiles><smiles>[3H]CC[3H]</smiles><smiles>[O-]OC1COCCO1</smiles>

$\downarrow \mathrm{O}_{2}$

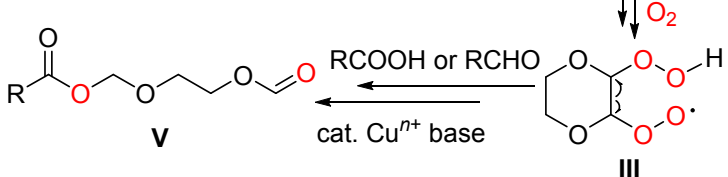

图式 $2 \mathrm{Cu}$ 催化二氧六环中 $\mathrm{C}\left(\mathrm{sp}^{3}\right)-\mathrm{C}\left(\mathrm{sp}^{3}\right)$ 单键氧化断裂可 能机理

Scheme 2 Mechanisms proposed for $\mathrm{Cu}$-catalyzed oxidative cleavage of the $\mathrm{C}\left(\mathrm{sp}^{3}\right)-\mathrm{C}\left(\mathrm{sp}^{3}\right)$ bond in dioxane

2013 年, Wang 课题组 ${ }^{[12]}$ 在可见光和 $\mathrm{Ru}$ 协同催化 下，成功地将一系列乙二胺类化合物通过 $\mathrm{C}\left(\mathrm{sp}^{3}\right)-$ 
$\mathrm{C}\left(\mathrm{sp}^{3}\right)$ 单键断裂转化为了甲酰胺, 该反应在氧气中室温 即可进行，条件温和(Eq. 4).

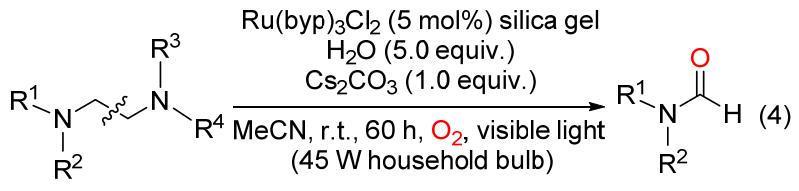

他们认为该反应可能按 Scheme 3 中的机理进行, 首先在可见光照射下 $\mathrm{Ru}(\mathrm{II})$ 被激活, 与底物 $\mathbf{I}$ 反应生成 不稳定的中间体 II, II 通过电子转移使 $\mathrm{C}\left(\mathrm{sp}^{3}\right)-\mathrm{C}\left(\mathrm{sp}^{3}\right)$ 单 键断裂并生成一个氨基自由基 III 和一个亚胺正离子 IV, III 可能被氧气捕获生成过氧化自由基 $\mathrm{V}, \mathrm{V}$ 可以作 为氧化剂将 $R u(I)$ 氧化为 $R u(I I)$ 实现催化剂循环, 同时生 成中间体 VI, VI 在碱作用下最终生成了甲酰胺.

2015 年, $\mathrm{Chiba}$ 课题组 ${ }^{[13]}$ 以 $\mathrm{CuCl}$ 为催化剂, 在氧气 和 $N$-差基邻苯二甲酰胺(NHPI)存在条件下, 以 1,1-二苯 基戊烷为原料一锅法合成了烯内酯. 他们认为原料在反 应条件下先生成半缩醛中间体, 然后再在该反应条件下 通过断裂 $\mathrm{C}\left(\mathrm{sp}^{3}\right)-\mathrm{C}\left(\mathrm{sp}^{3}\right)$ 单键生成内酯(Scheme 4$)$.

Chiba 等认为半缩醛中间体 I 生成后, 反应按照 Scheme 5 的机理进行: I 脱水生成烯醚 II, 自由基 PINO- 夺去 II 烯丙位上的氢生成 $\alpha$-氧自由基 III, III 失 去一个电子生成碳正离子 IV, IV 被 PINO - 自由基再夺 去一个氢原子生成环外烯烃 $\mathbf{V}, \mathbf{V}$ 与 $\mathrm{PINO}$ - 自由基结合 生成 $\alpha$-氧自由基 VI, VI 再被氧气氧化生成过氧化自由 基 VII, 最后通过电子转移使 $\mathrm{C}\left(\mathrm{sp}^{3}\right)-\mathrm{C}\left(\mathrm{sp}^{3}\right)$ 单键发生断 裂生成内酯 IX.

2012 年, van der Donk 课题组 ${ }^{[14]}$ 在氧气下, 使用甲 基磷酸酯酶 (MPnS) 催化 2-羟基乙基磷酸酯 (2-HEP) $\mathrm{C}\left(\mathrm{sp}^{3}\right)-\mathrm{C}\left(\mathrm{sp}^{3}\right)$ 单键氧化断裂生成甲基磷酸酯(MPn) (Eq. 5). 该反应是酶催化的反应, 酶中的金属 $\mathrm{Fe}(\mathrm{II})$ 在反应中 起着关键作用.

Donk 等认为 2-羟基乙基磷酸酯(2-HEP)与甲基磷酸

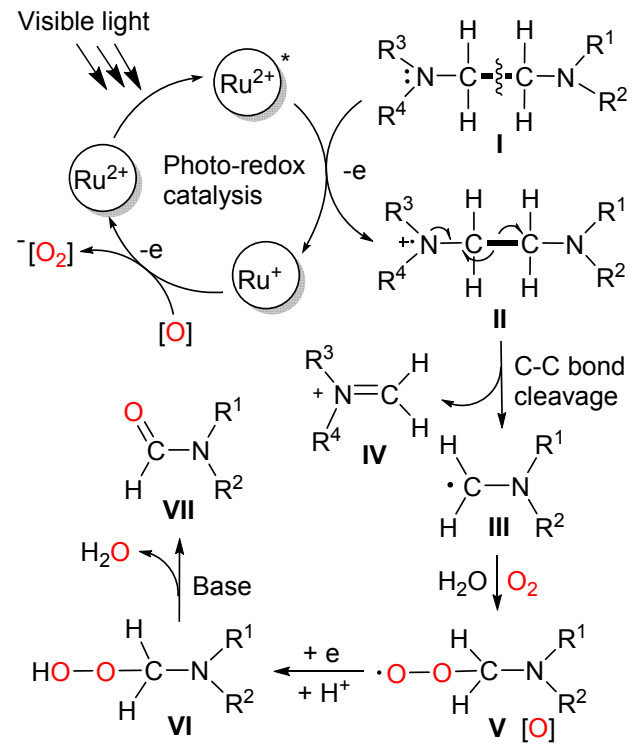

图式 3 可见光催化取代二乙胺 $\mathrm{C}\left(\mathrm{sp}^{3}\right)-\mathrm{C}\left(\mathrm{sp}^{3}\right)$ 单键断裂的可 能机理

Scheme 3 Mechanisms proposed for visible-light photo-catalyzed $\mathrm{C}\left(\mathrm{sp}^{3}\right)-\mathrm{C}\left(\mathrm{sp}^{3}\right)$ bond cleavage of substitutional diethylamine

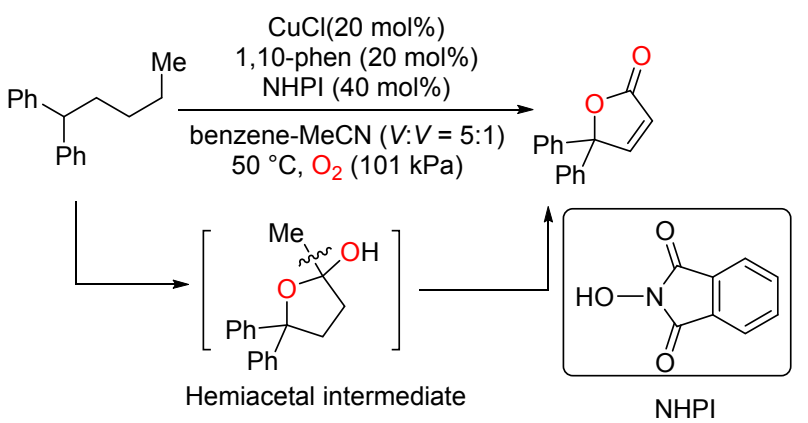

图式 $4 \mathrm{Cu}$ 催化 $\mathrm{C}\left(\mathrm{sp}^{3}\right)-\mathrm{C}\left(\mathrm{sp}^{3}\right)$ 单键断裂合成内酯 Scheme 4 Synthesis of lactone via $\mathrm{Cu}$-catalyzed $\mathrm{C}\left(\mathrm{sp}^{3}\right)-\mathrm{C}\left(\mathrm{sp}^{3}\right)$ bond cleavage

酯酶(MPn)结合后在氧气下得到 Fe(III)过氧化自由基 I, I 选择性夺去 2-HEP 上连有羟基碳上的一个氢原子生成

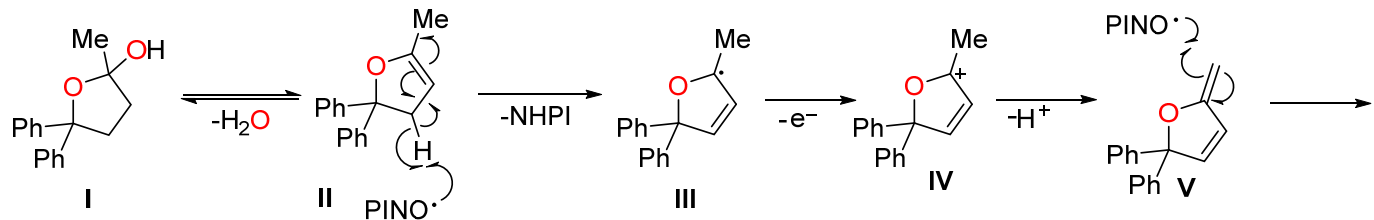

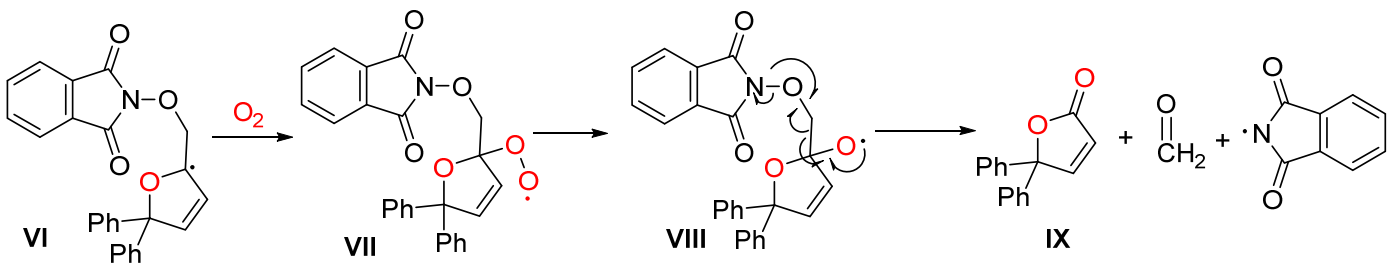

图式 $5 \mathrm{Cu}$ 催化 $\mathrm{C}\left(\mathrm{sp}^{3}\right)-\mathrm{C}\left(\mathrm{sp}^{3}\right)$ 单键氧化断裂合成内酯的反应可能机理

Scheme 5 Mechanisms proposed for synthesis of lactone via $\mathrm{Cu}$-catalyzed $\mathrm{C}\left(\mathrm{sp}^{3}\right)-\mathrm{C}\left(\mathrm{sp}^{3}\right)$ bond oxidative cleavage 


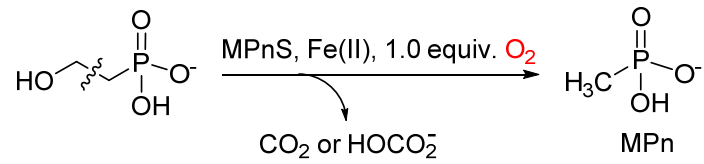

自由基负离子 II, II 将一个电子转移到 $\mathrm{Fe}(\mathrm{III})$ 上, 生成 与 $\mathrm{Fe}(\mathrm{III})$ 相连的膦酰基乙醛, 然后脱去一个 $\mathrm{H}^{+}$, 得到烷 基过氧化物中间体 III, III 中氧一氧键发生均裂生成二元 醇自由基, 再通过 $\mathrm{C}\left(\mathrm{sp}^{3}\right)-\mathrm{C}\left(\mathrm{sp}^{3}\right)$ 单键断裂, 生成中间体 IV, 该中间体的铁上连有一个 MPn 自由基和一个甲酸 盐, MPn 自由基夺去甲酸盐上的氢原子并通过电子转移 最终生成 MPn 和二氧化碳(Scheme 6).

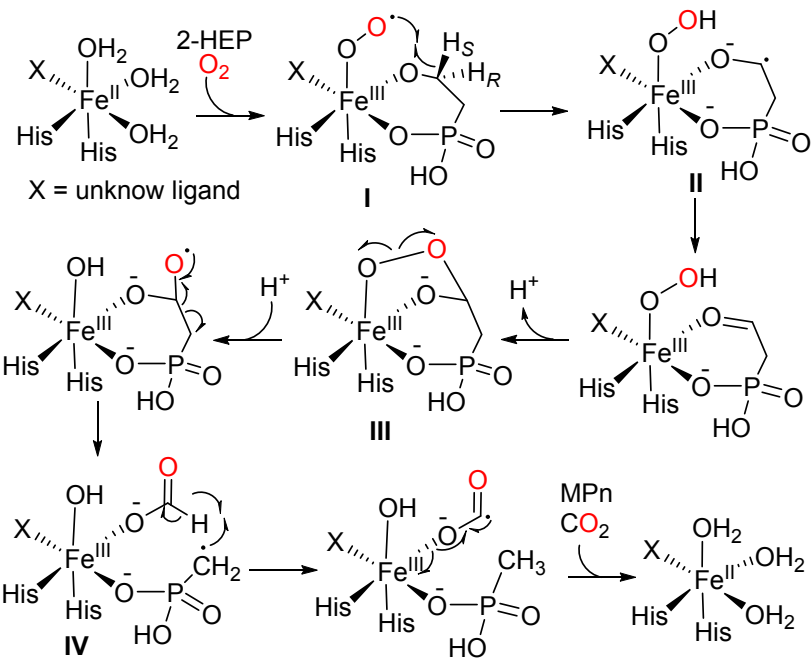

图式 6 甲基磷酸酯酶催化 $\mathrm{C}\left(\mathrm{sp}^{3}\right)-\mathrm{C}\left(\mathrm{sp}^{3}\right)$ 单键氧化断裂反应 可能机理

Scheme 6 Mechanisms proposed for methylphosphonate synthase catalyzed $\mathrm{C}\left(\mathrm{sp}^{3}\right)-\mathrm{C}\left(\mathrm{sp}^{3}\right)$ bond cleavage

2015 年, Loh 课题组 ${ }^{[15]}$ 报道了以空气中的氧气作为 唯一的氧化剂, $\operatorname{Pd}(\mathrm{OAc})_{2}$ 催化烯酮与苯肼反应生成二氢 吡唑中间体, 然后通过断裂 $\mathrm{C}\left(\mathrm{sp}^{3}\right)-\mathrm{C}\left(\mathrm{sp}^{3}\right)$ 单键一锅法 合成吡坐(Scheme 7). 该法提出了一种一锅法合成多取 代吡唑的新途径.
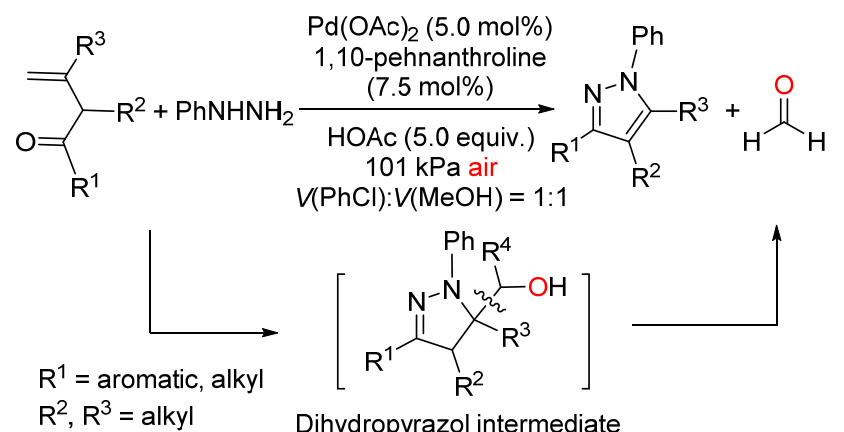

图式 7 Pd 催化的一锅法合成吡唑

Scheme 7 Pd catalyzed synthesis of pyrazole in one pot
Loh 等认为烯酮 I 先与苯肼缩合生成苯腙 II, II 在氧 气和 $\mathrm{Pd}(\mathrm{OAc})_{2}$ 催化下生成中间体 III, III 再与 $\mathrm{Pd}$ 作用生 成中间体 IV, IV 通过 $\beta$-碳和 $\beta$-氢两步消除得到目标化 合物 VI (Scheme 8).
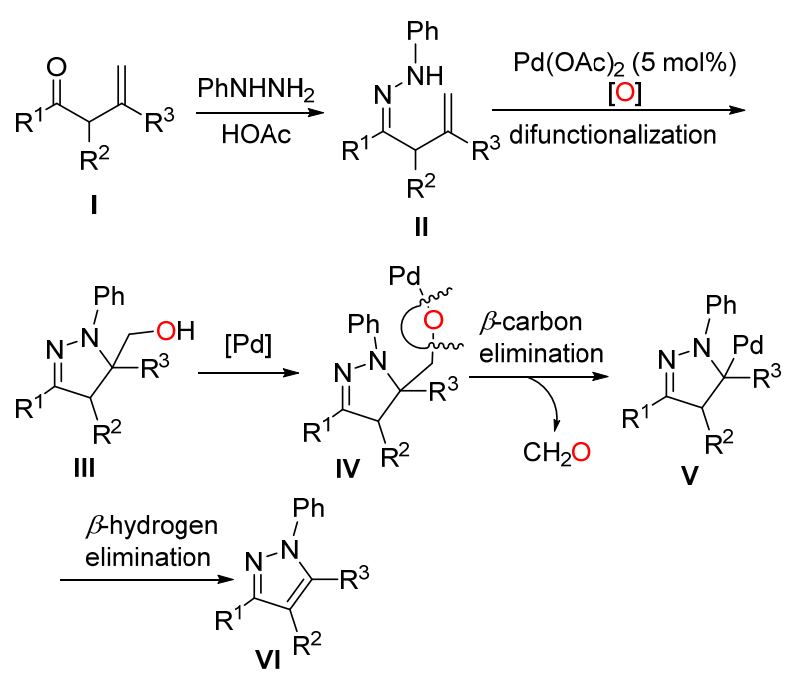

图式 $8 \mathrm{Pd}$ 催化 $\mathrm{C}\left(\mathrm{sp}^{3}\right)-\mathrm{C}\left(\mathrm{sp}^{3}\right)$ 单键氧化断裂反应的可能机理 Scheme 8 Mechanisms proposed for Pd catalyzed $\mathrm{C}\left(\mathrm{sp}^{3}\right)^{3}$ $\mathrm{C}\left(\mathrm{sp}^{3}\right)$ oxidative cleavage

\section{$1.2 \mathrm{C}\left(\mathrm{sp}^{2}\right)(\mathrm{CO})-\mathrm{C}\left(\mathrm{sp}^{3}\right)$ 单键断裂反应}

由于羰基的吸电子作用, 使得 $\mathrm{C}\left(\mathrm{sp}^{2}\right)(\mathrm{CO})-\mathrm{C}\left(\mathrm{sp}^{3}\right)$ 单键的活性明显比无羰基 $\mathrm{C}\left(\mathrm{sp}^{3}\right)-\mathrm{C}\left(\mathrm{sp}^{3}\right)$ 单键要高, 近 年来碳一碳单键的断裂反应研究也是主要针对该类型键, 并取得了较大的进展，所以本文也只对含有羰基的 $\mathrm{C}\left(\mathrm{sp}^{2}\right)-\mathrm{C}\left(\mathrm{sp}^{3}\right)$ 单键的断裂反应进行了综述，根据断裂 反应生成不同的产物, 分为六类分别进行阐述.

\subsection{1 $\mathrm{C}\left(\mathrm{sp}^{2}\right)(\mathrm{CO})$ - $\mathrm{C}\left(\mathrm{sp}^{3}\right)$ 单键断裂生成醛}

2013 年, $\mathrm{Bi}$ 和 $\mathrm{Liu}$ 课题组 ${ }^{[16]}$ 首次报道了氧气下, $\mathrm{CuI}$ 催化的芳香酮或脂肪酮选择性氧化断裂 $\mathrm{C}\left(\mathrm{sp}^{2}\right)(\mathrm{CO})$ $\mathrm{C}$ (methyl)单键并成醛(Eq. 6). 当羰基酮的 $\alpha$ 位不是甲基 而是其它脂肪链时反应也可以发生, 但需要适当延长反 应时间. 该反应的最大优点就是生成的氧化产物停留在 醛的阶段而不会被过度氧化, 所以该反应提供了一种新 颖的将甲基酮转化为醛的好方法.

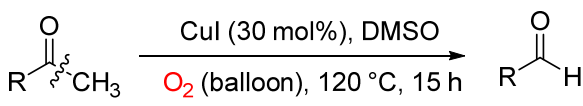

$$
\begin{aligned}
& \mathrm{R}=(\mathrm{Het}) \mathrm{Ar}, \text { Alkyl }
\end{aligned}
$$

Liu 等认为该反应机理按照 Scheme 9 进行. 首先底 物 I 在 $\mathrm{Cu}(\mathrm{I})$ 催化下被氧气氧化生成 $\alpha$-羟基苯乙酮 II, 然 后进一步被氧化，脱去一分子水生成苯甲酰甲醛中间体 III, III 被一分子水亲核进攻生成 $\alpha, \alpha$-二羟基苯乙酮 IV, 再通过类似于 Cannizzaro 反应进行 1,2-氢转移得到中间 
体 $\mathbf{V}$, 最后通过电子转移使 $\mathrm{C}\left(\mathrm{sp}^{2}\right)(\mathrm{CO})-\mathrm{C}\left(\mathrm{sp}^{3}\right)$ 单键断 裂生成目标化合物 $\mathrm{VI}$, 释放出一分子甲酸, 甲酸在 $\mathrm{Cu}(\mathrm{II})$ 催化下生成二氧化碳和氢气，与此同时 $\mathrm{Cu}(\mathrm{II})$ 转 变为 $\mathrm{Cu}(\mathrm{I})$ 实现催化剂循环.

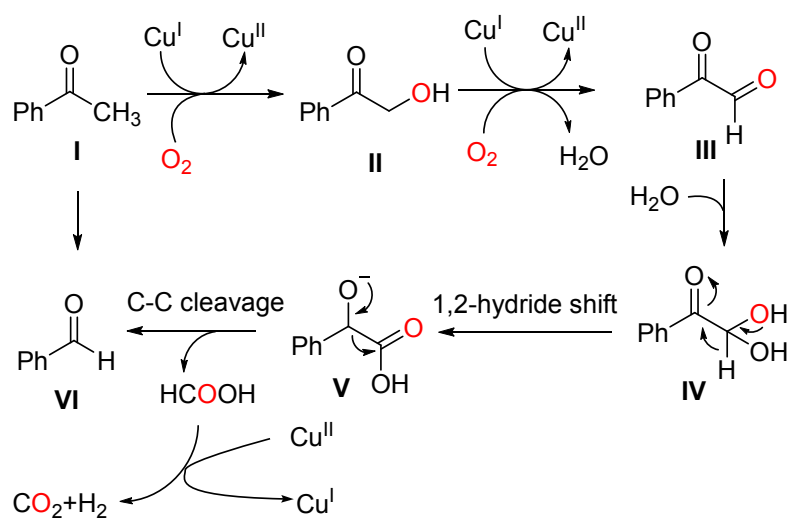

图式 $9 \mathrm{Cu}$ 催化酮生成醛反应的可能机理

Scheme 9 Mechanisms proposed for $\mathrm{Cu}$-catalyzed synthesis of aldehyde from ketone

2015 年, Zhou 和 Jiao 课题组 ${ }^{[17]}$ 使用 $\mathrm{CuCl}_{2} \cdot 2 \mathrm{H}_{2} \mathrm{O}$ 作 为催化剂, 在胺和空气存在下, 成功地将无张力环酮的 $\mathrm{C}\left(\mathrm{sp}^{2}\right)(\mathrm{CO})-\mathrm{C}\left(\mathrm{sp}^{3}\right)$ 单键氧化断裂生成分子内含有酰胺 的醛(Eq. 7). 该反应能一步生成碳-氧双键和碳一氮单键, 他们使用该方法合成了 36 个收率最高达 $93 \%$ 的目标化 合物.

$$
\begin{aligned}
& \int_{\mathrm{R}}+\mathrm{HNR}^{1} \mathrm{R}^{2} \frac{\begin{array}{l}
\mathrm{CuCl}_{2} \cdot 2 \mathrm{H}_{2} \mathrm{O}(10 \mathrm{~mol} \%) \\
1,10-\text { phen }
\end{array} \mathrm{H}_{2} \mathrm{O}(2 \mathrm{~mol} \%)}{\mathrm{CH}_{3} \mathrm{CN} / \text { dioxane }(\mathrm{VIV}=3 / 1)} \\
& \overbrace{}^{\mathrm{H}} \cdots{ }_{\mathrm{N}}^{\mathrm{O}}
\end{aligned}
$$

$\mathrm{Jiao}$ 等认为 $\mathbf{I}$ 先与酮在氧气下生成 $\mathrm{Cu}(\mathrm{III})$ 过氧化自 由基 II, II 异构化生成过氧化合物 III, III 与胺发生亲核 反应得到过氧化中间体 IV, IV 发生均裂得到 $\mathrm{Cu}(\mathrm{II})$ 化合 物 $\mathbf{V}$ 和中间体 VI, VI 发生可逆的 $\beta$ 裂解得到中间体 VII，VII 进一步被中间体 V 氧化得到目标化合物 VIII 同时释放 VI, VI 在 $\mathrm{HCl}$ 的辅助下实现催化剂循环 (Scheme 10).

2016 年, $\mathrm{Li}$ 课题组 ${ }^{[18]}$ 使用更廉价的 $\mathrm{FeCl}_{3}$ 作为催化 剂, 在空气中催化的 1-芳基-丙-2-酮类化合物的 $\mathrm{C}\left(\mathrm{sp}^{2}\right)$ $(\mathrm{CO})-\mathrm{C}\left(\mathrm{sp}^{3}\right)$ 单键发生氧化断裂生成碳链缩短的醛、酮 或 1,2-二羰基化合物的反应(Eq. 8), 该反应可用于合成 2-乙酰氨基-苯甲醛类化合物, 这类化合物是合成喹啉$2(1 H)$-酮原料.

$\mathrm{Li}$ 等认为该反应的机理是底物酮首先与 $\mathrm{Fe}(\mathrm{III})$ 结合

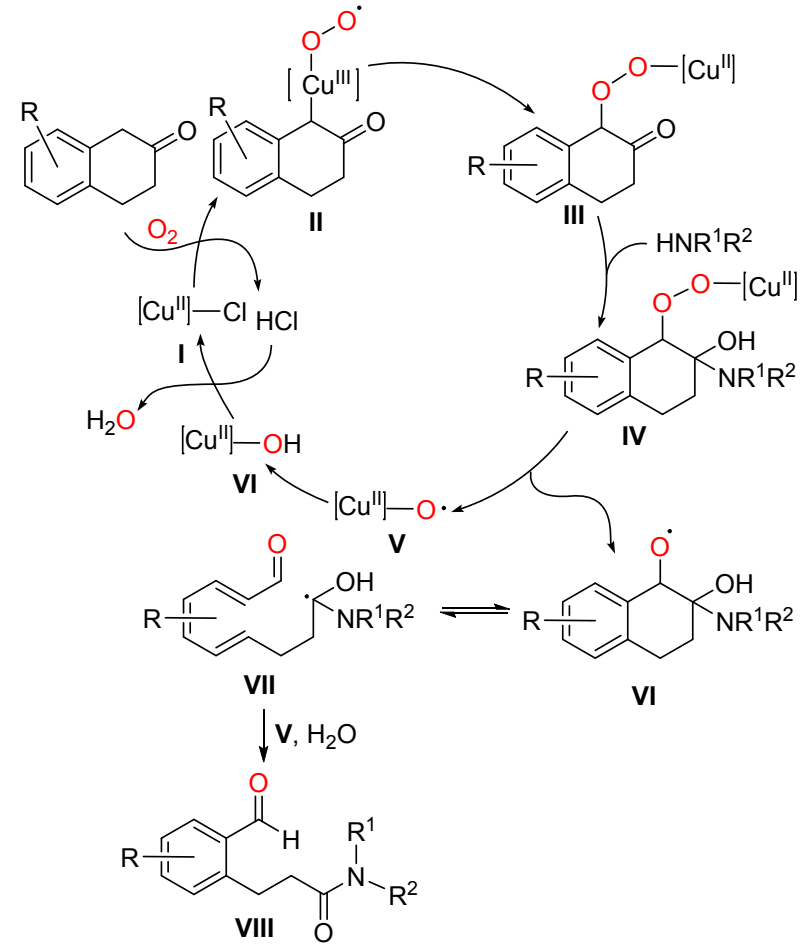

图式 $10 \mathrm{Cu}$ 催化无张力环酮生成醛的可能反应机理 Scheme 10 Mechanisms proposed for $\mathrm{Cu}$-catalyzed synthesis of aldehydes from unstrained cyclic ketones

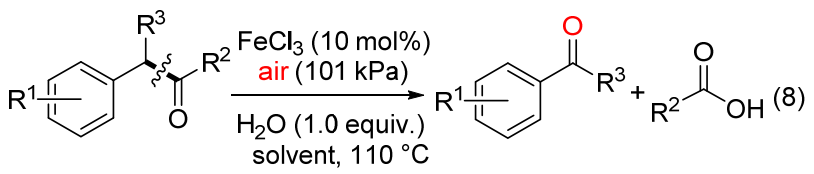

生成铁-烯醇化合物 $\mathbf{I}, \mathbf{I}$ 捕获一分子氧气生成过氧化物 II，其共振式 III 与水分子亲核反应得到过氧化物中间 体 IV, IV 通过电子转移, 断裂 $\mathrm{C}\left(\mathrm{sp}^{2}\right)(\mathrm{CO})-\mathrm{C}\left(\mathrm{sp}^{3}\right)$ 单键, 得到目标化合物醛同时释放出一分子酸，脱下的 $\mathrm{Fe}(\mathrm{OH}) \mathrm{Cl}_{2}$ 与 $\mathrm{HCl}$ 结合脱水后得到 $\mathrm{FeCl}_{3}$, 实现催化剂循 环(Scheme 11).

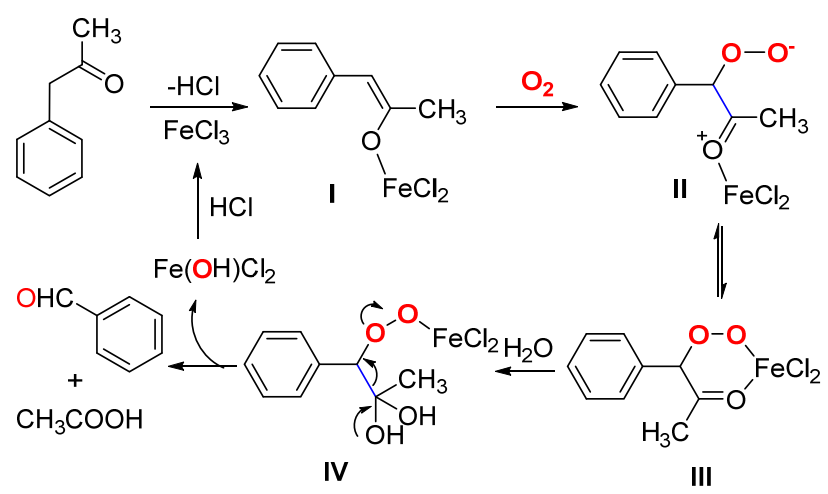

图式 $11 \mathrm{Fe}$ 催化酮生成碳链缩短醛反应的可能机理 Scheme 11 Mechanisms proposed for Fe-catalyzed synthesis of carbon chain-shortened aldehydes from ketones 


\subsection{2 $\mathrm{C}\left(\mathrm{sp}^{2}\right)(\mathrm{CO})-\mathrm{C}\left(\mathrm{sp}^{3}\right)$ 单键断裂生成酮}

2012 年, Wang 课题组 ${ }^{[19]}$ 利用 Rh 的螯合作用, 在空 气中成功将酮的甲基或芳基直接置换成另外一个芳基 (Eq. 9), 新的芳基来源于芳基硼酸, 该反应中导向基团 在反应中起着关键作用.

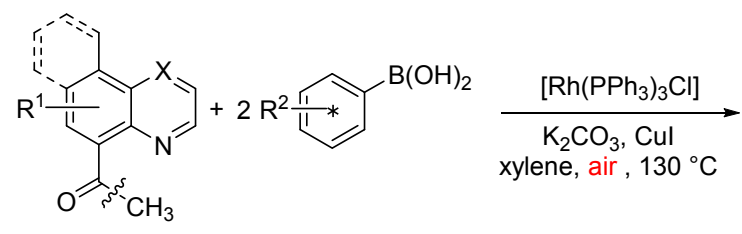<smiles></smiles>

(9)

Wang 等认为该反应可能经由 Scheme 12 的机理过 程: 首先 $\mathrm{Rh}(\mathrm{I})$ 在定位基团的辅助下螯合到底物芳乙酮 乙酰基的碳-碳单键上, 形成 $\mathrm{Rh}(\mathrm{III})$ 的五元环中间体 I, I 与芳基硼酸发生转金属化得到 $\mathrm{Rh}$ 上连有甲基和芳基中 间体 II, II 插入一个磷配体, 接着芳基和甲基发生消除 得到 $\mathrm{Rh}(\mathrm{I})$ 中间体 III, 在氧气下, $\mathrm{Cu}(\mathrm{I})$ 将 III 中的 $\mathrm{Rh}(\mathrm{I})$ 氧化到 Rh(III)生成中间体 IV, 中间体 IV 再一次与芳基 嗍酸发生转金属化得到中间体 $\mathbf{V}, \mathbf{V}$ 通过还原消除得到 目标产物和 $\mathrm{Rh}(\mathrm{I})$, 从而实现催化剂循环.

2013 年, $\mathrm{Xia}$ 课题组 ${ }^{[20]}$ 使用 $\mathrm{Ru}(\mathrm{bpy})_{3} \mathrm{Cl}_{2}$ 作为光 催化剂, 在光照、胺和空气存在下将醛 $\mathrm{C}\left(\mathrm{sp}^{2}\right)(\mathrm{CO})$ $\mathrm{C}\left(\mathrm{sp}^{3}\right)$ 单键氧化断裂, 以较高的收率得到酮和甲酰胺 (Scheme 13). 该反应在空气中室温就可以进行, 反应条 件比较温和.

$\mathrm{Xia}$ 等认为醛首先和胺反应生成烯胺 $\mathbf{I}$, 接着烯胺 $\mathbf{I}$ 被光激活的 $\mathrm{Ru}(\mathrm{II})$ 氧化为阳离子自由基 II 和 $\mathrm{Ru}(\mathrm{I})$, $\mathrm{Ru}(\mathrm{I})$ 被氧气氧化为 $\mathrm{Ru}(\mathrm{II})$, 使催化剂循环, 自由基 II 与 $\left[\mathrm{O}_{2}\right] \bullet-$ 作用生成 1,2-二氧杂环丁烷中间体, 最后通过电 子转移使 $\mathrm{C}\left(\mathrm{sp}^{2}\right)(\mathrm{CO})-\mathrm{C}\left(\mathrm{sp}^{3}\right)$ 单键断裂, 生成目标产物 酮和甲酰胺(Scheme 14).

2014 年, Huang 课题组 ${ }^{[21]}$ 报道了在氧气和四氢吡 咯存在条件下, $\mathrm{Au}$ 催化醛 $\mathrm{C}\left(\mathrm{sp}^{2}\right)(\mathrm{CO})-\mathrm{C}\left(\mathrm{sp}^{3}\right)$ 单键断裂 并与高碘化合物 TIPS-EBX 反应生成炔酮(Eq. 10).

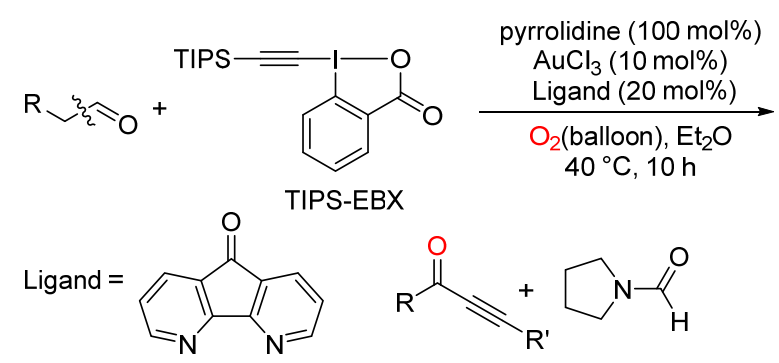

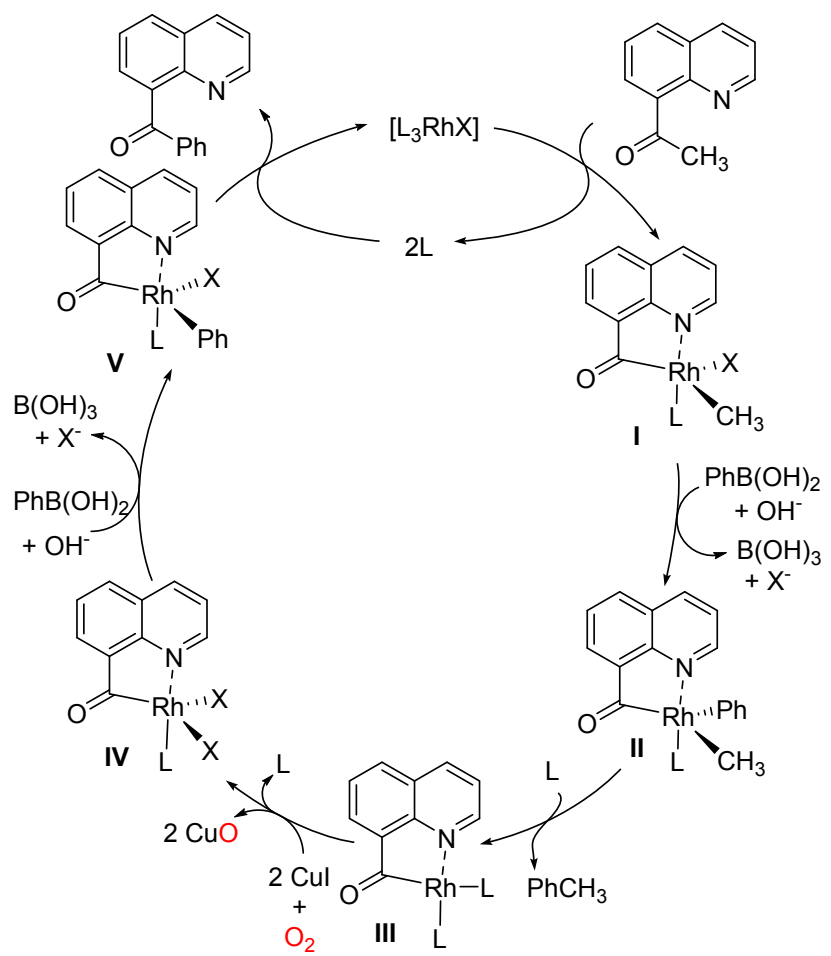

图式 $12 \mathrm{Rh}$ 和 $\mathrm{Cu}$ 协同催化芳乙酮 $\mathrm{C}\left(\mathrm{sp}^{2}\right)(\mathrm{CO})-\mathrm{C}\left(\mathrm{sp}^{3}\right)$ 键断裂 可能机理

Scheme 12 Mechanisms proposed for $\mathrm{Rh}$ and $\mathrm{Cu}$ synergetic catalyzed $\mathrm{C}\left(\mathrm{sp}^{2}\right)(\mathrm{CO})-\mathrm{C}\left(\mathrm{sp}^{3}\right)$ cleavage of aromatic ethyl ketone

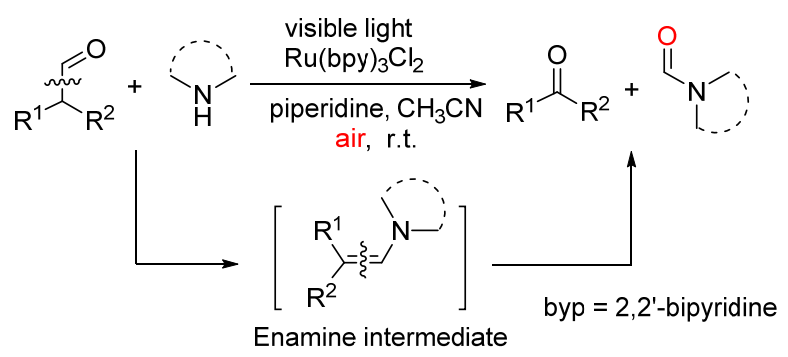

图式 $13 \mathrm{Ru}$ 和可见光催化合成酮反应

Scheme $13 \mathrm{Ru}$ and visible-light photoredox catalyzed synthesis of ketones

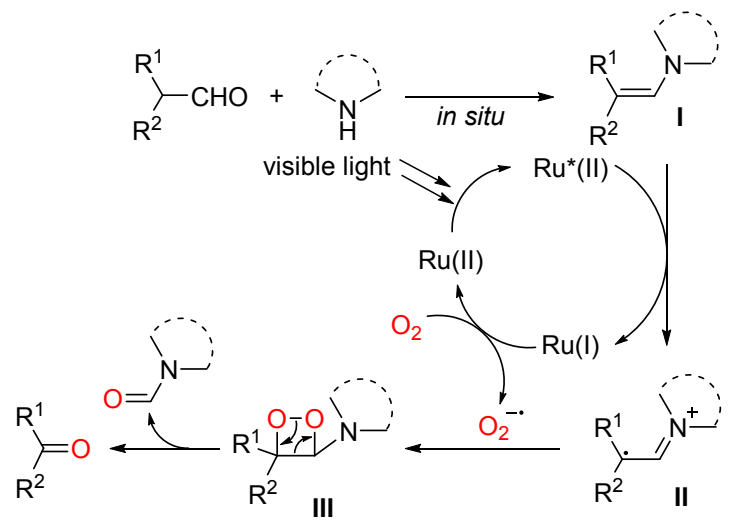

图式 $14 \mathrm{Ru}$ 和可见光催化合成酮反应的可能机理

Scheme 14 Mechanisms proposed for $\mathrm{Ru}$ and visible-light photoredox catalyzed synthesis of ketones 
Huang 等认为酫 I 在 $\mathrm{Au}(\mathrm{I})$ 或 $\mathrm{Au}(\mathrm{III})$ 催化下与 TIPS-EBX 反应得到连烯醛 II, II 与四氢吡咯反应生成 炔烯胺中间体 III, III 在 $\mathrm{Au}(\mathrm{III})$ 催化下进一步与氧气发 生自由基反应生成 1,2-二氧杂环丁烷中间体 IV, IV 通过 $\mathrm{C}\left(\mathrm{sp}^{2}\right)(\mathrm{CO})-\mathrm{C}\left(\mathrm{sp}^{3}\right)$ 单键断裂生成目标化合物 $\mathbf{V}$ 同时释 放副产物 VI (Scheme 15).

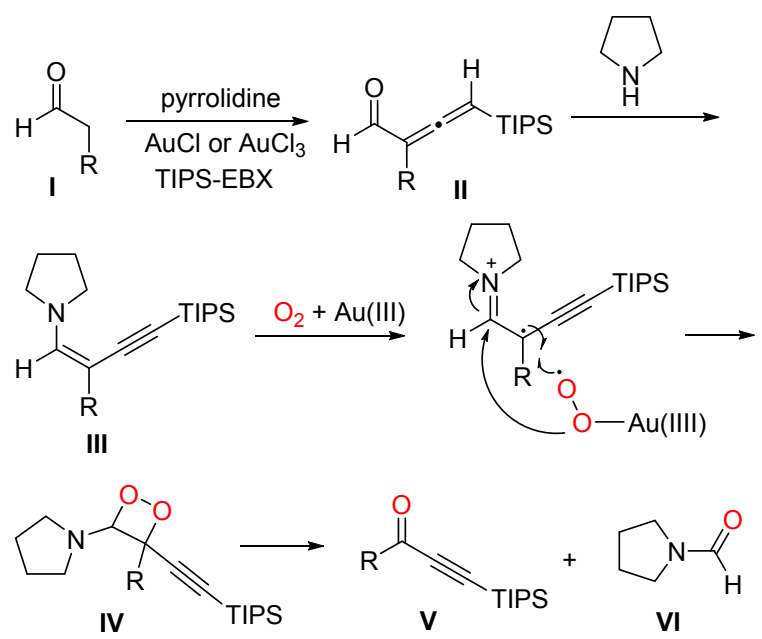

图式 $15 \mathrm{Au}$ 催化由醛合成炔酮反应的可能机理

Scheme 15 Mechanisms proposed for Au-catalyzed synthesis of ynones from aldehydes

2014 年, Jiao 课题组 ${ }^{[22]}$ 使用 $\mathrm{CuBr}$ 作为催化剂, 在 氧气下催化 1,3 -二芳基二酮 $\mathrm{C}\left(\mathrm{sp}^{2}\right)(\mathrm{CO})-\mathrm{C}\left(\mathrm{sp}^{3}\right)$ 单键断 裂生成 1,2-二酮(Eq. 11), 反应的结果是将 1,3-二芳基二 酮类化合物中的亚甲基碳切除, 生成直接将两个羰基碳 用碳一碳单键相连的产物.

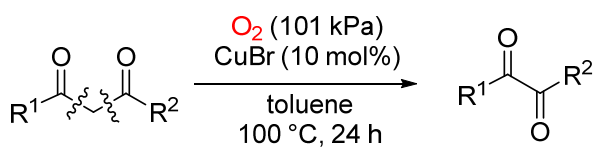

Jiao 等提出如 Scheme 16 所示的机理: $\mathrm{Cu}(\mathrm{I})$ 在氧气 下氧化成 $\mathrm{Cu}(\mathrm{II}), \mathrm{Cu}(\mathrm{II})$ 与底物 $\mathbf{I}$ 发生催化氧化反应生成 关键中间体 II, II 在 $\mathrm{Cu}(\mathrm{II})$ 催化下发生类似于 1,2Wagner-Meerwein 重排反应得到中间体 III, III 通过电子 转移使 $\mathrm{C}\left(\mathrm{sp}^{2}\right)(\mathrm{CO})-\mathrm{C}\left(\mathrm{sp}^{3}\right)$ 单键断裂生成目标产物, 并 释放出一氧化碳.

2014 年, Maiti 课题组 ${ }^{[23]}$ 报道了氧气下 $\mathrm{Cu}(\mathrm{OAc})_{2}$ 催 化将二芳基乙酮苄位碳切除生成二芳基甲酮的方法(Eq. 12). 该反应除了一个茮基碳被切除之外, 其它结构保 持不变.

对于该反应, Maiti 等提出如 Scheme 17 的机理, 他 们认为底物 I 首先与胺生成亚胺 II, 接着在 $\mathrm{Cu} / \mathrm{O}_{2}$ 催化 系统下苄基碳被氧化生成对应的酮 III, III 在 $\mathrm{Cu}(\mathrm{II})$ 辅助 下通过二苯乙醇酸重排得到目标化合物 IX.

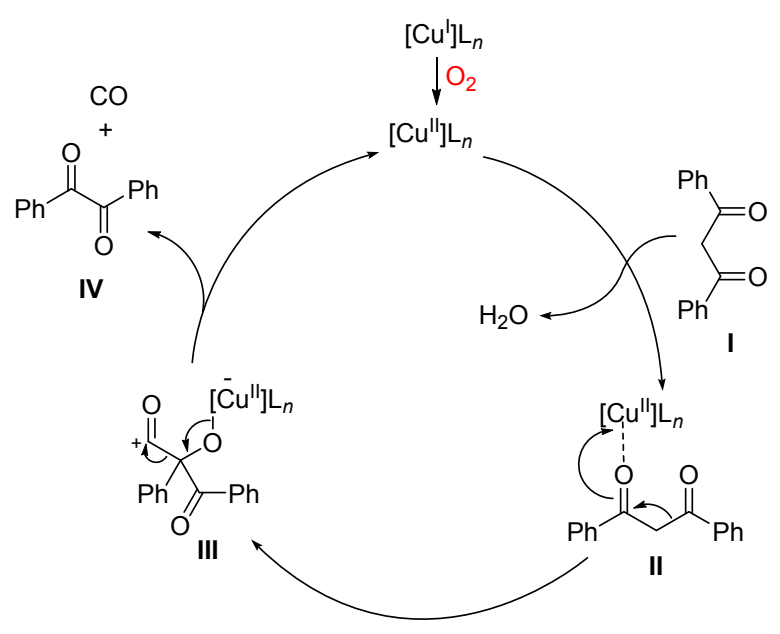

图式 $16 \mathrm{CuBr}$ 催化 1,3-二芳基二酮合成 1,2-二芳基酮反应的 可能机理

Scheme 16 Mechanisms proposed for $\mathrm{CuBr}$ catalyzed synthesis of 1,2-diarylketones from 1,3-diaryldiketones
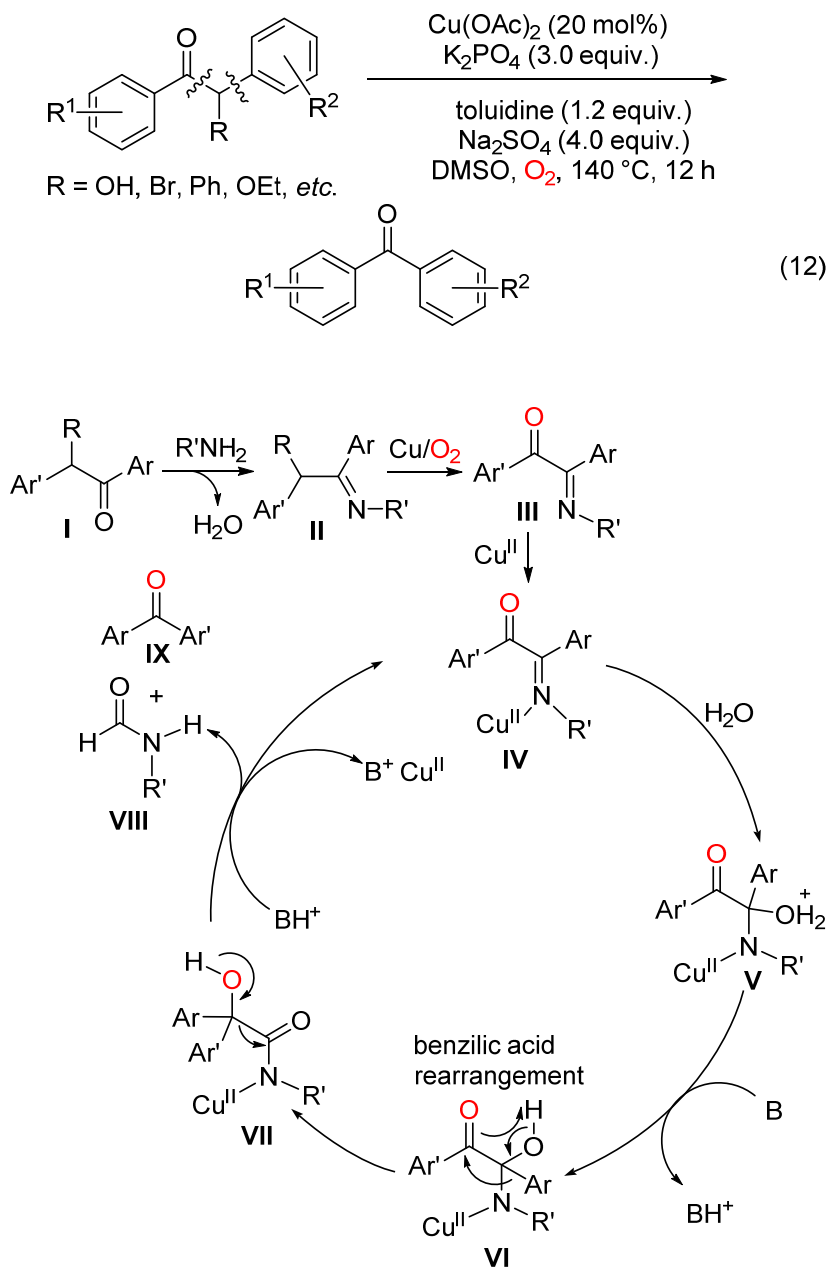

图式 $17 \mathrm{Cu}(\mathrm{OAc})_{2}$ 催化通过切除苄位碳合成二芳基甲酮反应 的可能机理

Scheme 17 Mechanisms proposed for $\mathrm{Cu}(\mathrm{OAc})_{2}$ catalyzed synthesis of diaryl ketones by removal of benzylic carbon 
2013 年, $\mathrm{Fu}$ 课题组 ${ }^{[24]}$ 以 $\mathrm{Cu}\left(\mathrm{O}_{2} \mathrm{CCF}_{3}\right)_{2}$ 为催化剂, 氧 气下催化 2-芳胺基苯乙酮发生分子内的 $\mathrm{C}\left(\mathrm{sp}^{2}\right)(\mathrm{CO})$ $\mathrm{C}\left(\mathrm{sp}^{3}\right)$ 单键和碳-氢键活化断裂生成叮啶酮(Eq. 13). 该 方法可较好收率地合成带有多种官能团的呋啶酤类化 合物.

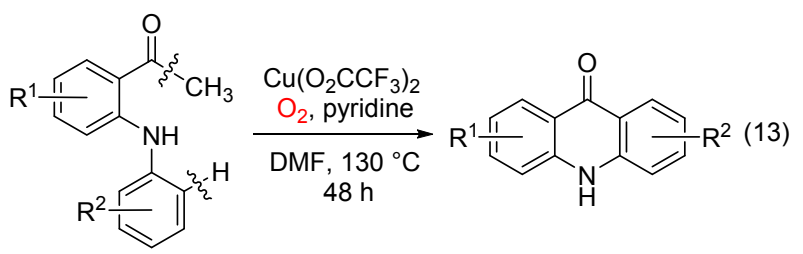

$\mathrm{Fu}$ 等认为首先底物在 $\mathrm{Cu}(\mathrm{II})$ 催化下被氧气氧化成 1-芳基二氢吲哚-2,3-二酮 $\mathbf{I}$, 加热条件下 I 在吡啶和水中 生成中间体 II, II 通过傅-克酰基化反应关环得到中间 体 III, III 脱水得到中间体 IV, 中间体 IV 脱去二氧化碳 和吡啶得到中间体 $\mathbf{V}, \mathbf{V}$ 在 Subramanian $\mathrm{Cu}$ 的催化下被 氧化生成 VI, VI共振后得到目标化合物 VII. 化合物 III 在 $\mathrm{Cu}(\mathrm{II})$ 的催化下脱去吡啶得到目标化合物 VII 是另外 一种可能的途径(Scheme 18).

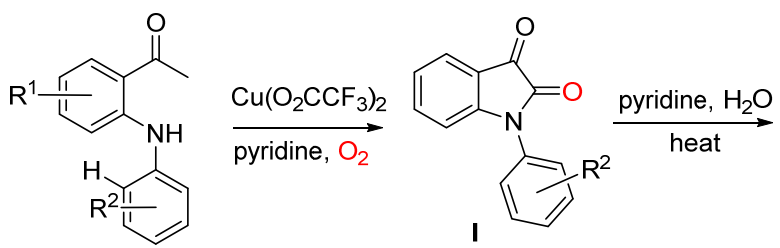

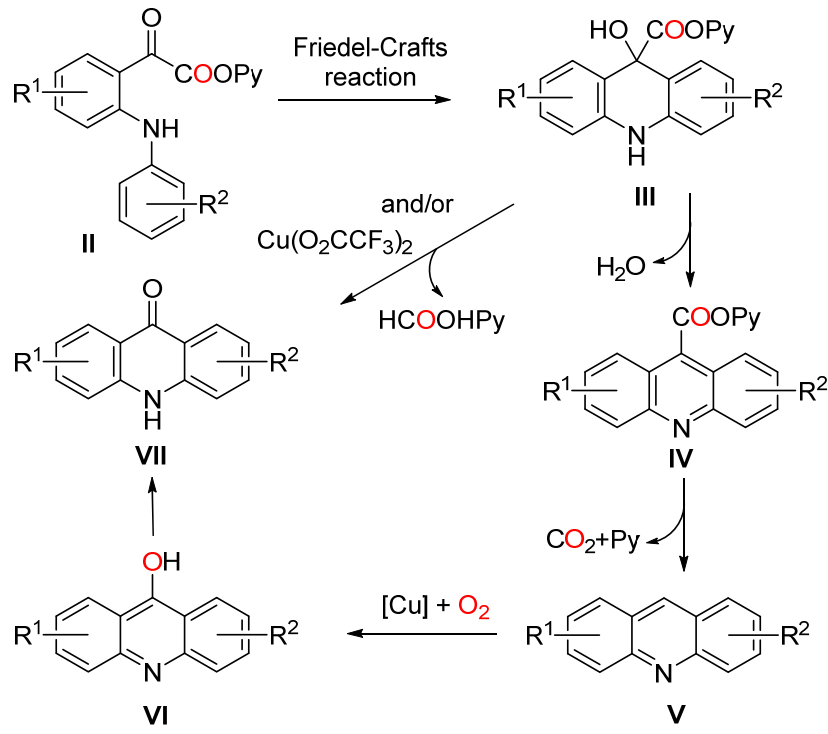

图式 $18 \mathrm{Cu}\left(\mathrm{O}_{2} \mathrm{CCF}_{3}\right)_{2}$ 催化 $\mathrm{C}\left(\mathrm{sp}^{2}\right)(\mathrm{CO})-\mathrm{C}\left(\mathrm{sp}^{3}\right)$ 断裂合成叮啶 酮反应的可能机理

Scheme 18 Mechanisms proposed for $\mathrm{Cu}\left(\mathrm{O}_{2} \mathrm{CCF}_{3}\right)_{2}$ catalyzed synthesis of acridone by cleavage of $\mathrm{C}\left(\mathrm{sp}^{2}\right)(\mathrm{CO})-\mathrm{C}\left(\mathrm{sp}^{3}\right)$

1.2.3 $\mathrm{C}\left(\mathrm{sp}^{2}\right)(\mathrm{CO})-\mathrm{C}\left(\mathrm{sp}^{3}\right)$ 单键断裂生成酸或酸酐 2011 年, $\mathrm{Li}$ 课题组 ${ }^{[25]}$ 以 $\mathrm{Cu}$ 盐为催化剂, 在氧气下
催化 $\alpha$-氨基羰基化合物 $\mathrm{C}\left(\mathrm{sp}^{2}\right)(\mathrm{CO})-\mathrm{C}\left(\mathrm{sp}^{3}\right)$ 单键断裂生 成相应的酸和甲酰胺(Eq. 14). 该反应使用附着在 $\mathrm{Fe}$ 上 $\mathrm{Cu}$ 作为固相催化剂, 反应后只需过滤就可以将催化剂 回收.

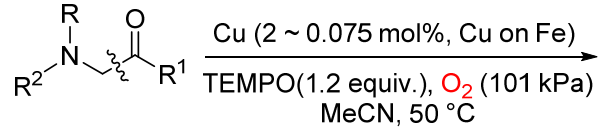

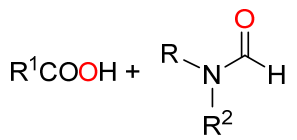

$\mathrm{Li}$ 等认为由 $\mathrm{Cu}$ 和氧气反应得到中间体 $\mathbf{I}, \mathbf{I}$ 与亚胺 中间体 III 发生加成反应得到中间体 IV, IV 发生 $\mathrm{C}\left(\mathrm{sp}^{2}\right)(\mathrm{CO})-\mathrm{C}\left(\mathrm{sp}^{3}\right)$ 键断裂和还原消除反应得到中间体 $\mathbf{V}, \mathbf{V}$ 通过氧一氧键断裂以及还原消除得到目标化合物 $\mathbf{V I}$ 和苯甲酸(Scheme 19).

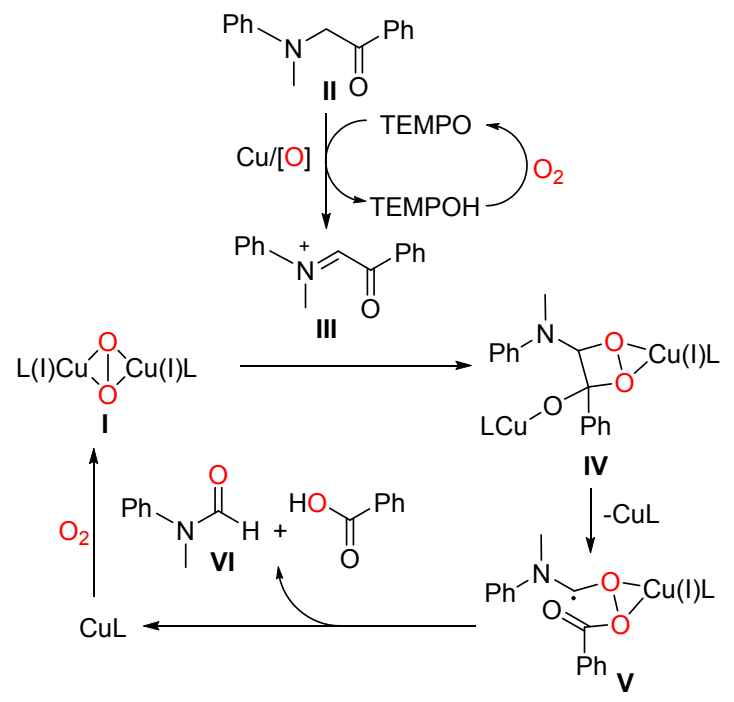

图式 $19 \mathrm{Cu}$ 催化 $\alpha$-氨基羰基化合物 $\mathrm{C}\left(\mathrm{sp}^{2}\right)(\mathrm{CO})-\mathrm{C}\left(\mathrm{sp}^{3}\right)$ 键断 裂反应的可能机理

Scheme 19 Mechanisms proposed for Cu-catalyzed cleavage of $\mathrm{C}\left(\mathrm{sp}^{2}\right)(\mathrm{CO})-\mathrm{C}\left(\mathrm{sp}^{3}\right)$ bond in $\alpha$-aminocarbonyl compounds

2012 年, Paine 课题组 ${ }^{[26]}$ 报道了使用 2,4'-二羟基苯 乙酮双加氧酶(DAD), 在氧气下, 将 $2,4^{\prime}$-二羟基苯乙酮 中的 $\mathrm{C}\left(\mathrm{sp}^{2}\right)(\mathrm{CO})-\mathrm{C}\left(\mathrm{sp}^{3}\right)$ 单键氧化断裂生成对羟基苯甲 酸和甲酸, 氧气分子中的两个氧原子分别转化到两个产 物中(Eq. 15).

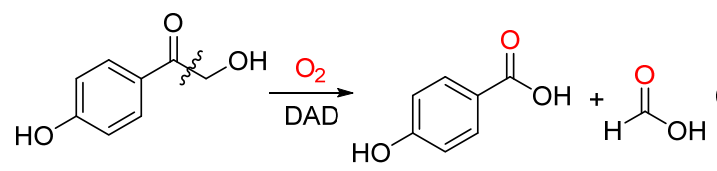

Paine 等认为 DAD 与 2,4'-二羟基苯乙酮的复合物 I 与分子氧生成 $\mathrm{Fe}(\mathrm{III})$ 过氧化自由基 II, II 从羟基酮的 $\alpha$ 
位夺取一个氢原子, 生成中间体 III, III 中 Fe(III)上的过 氧化氢迁移得到过氧化氢中间体 IV, IV 再生成 1,2-二氧 杂环丁烷中间体, 并最终断裂 $\mathrm{C}\left(\mathrm{sp}^{2}\right)(\mathrm{CO})-\mathrm{C}\left(\mathrm{sp}^{3}\right)$ 单键 和氧一氧单键生成产物(Scheme 20).
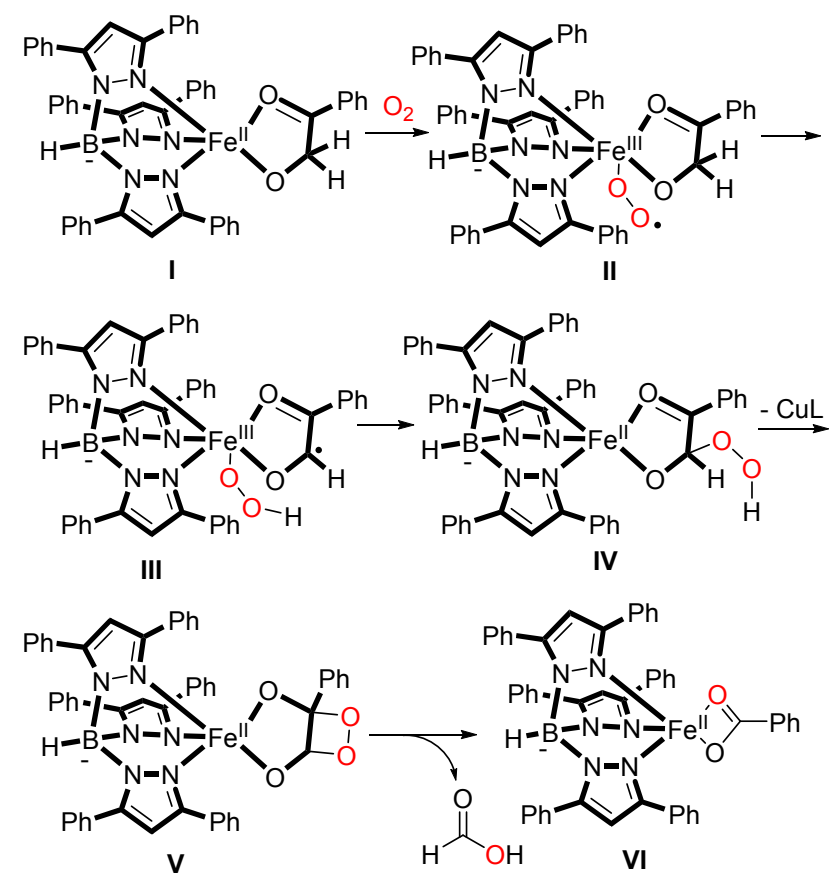

图式 $20 \mathrm{DAD}$ 催化的 2,4'-二羟基苯乙酮 $\mathrm{C}\left(\mathrm{sp}^{2}\right)(\mathrm{CO})-\mathrm{C}\left(\mathrm{sp}^{3}\right)$ 单键氧化断裂反应的可能机理

Scheme 20 Mechanisms proposed for DAD-catalyzed cleavage of the $\mathrm{C}\left(\mathrm{sp}^{2}\right)(\mathrm{CO})-\mathrm{C}\left(\mathrm{sp}^{3}\right)$ bond in 2,4'-dihydroxyacetophenone

2015 年, Bathula 课题组 ${ }^{[27]}$ 使用 $\mathrm{Cu} / \mathrm{O}_{2}$ 催化系统在 羟胺存在条件下成功地将酮转化成了酸(Eq. 16). 该反 应的底物可以是芳基酮、芳烷基酮或乙烯酮， $\mathrm{R}$ 基团可 以是甲基, 也可以是其它脂肪链, 但碳一碳单键断裂的 位置相同，即该反应具有一定的化学选择性.

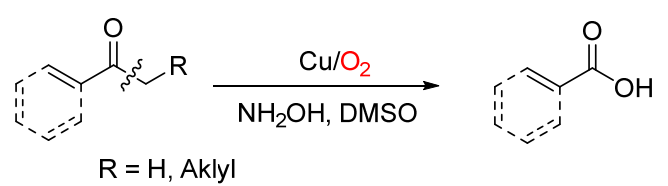

Bathula 等认为该反应可能经由下 Scheme 21 机理: 酮先与羟胺反应生成肟 $\mathbf{I}$, 接着在 $\mathrm{Cu}(\mathrm{I})$ 催化下被氧化成 $\alpha$-醛基肜 II, II 再与羟胺反应形成二肜化合物 III, III 脱 水生成氰基苯甲酰肪 $\mathbf{I V}, \mathbf{I V}$ 再通过 $\mathrm{C}\left(\mathrm{sp}^{2}\right)(\mathrm{CO})-\mathrm{C}\left(\mathrm{sp}^{3}\right)$ 单键断裂, 脱去一分子氢氧酸, 得到中间体 $\mathbf{V}, \mathbf{V}$ 与水加 成反应得到 VI, VI 最后脱去一分子氨气得到目标化合 物酸.

2016 年, Schoenebeck 课题组 ${ }^{[28]}$ 使用 $\mathrm{Cu}_{2} \mathrm{O}$ 在碱 $\mathrm{hppH}$ 和氧气下，催化环酮发生分子内 $\mathrm{C}\left(\mathrm{sp}^{2}\right)(\mathrm{CO})$ -

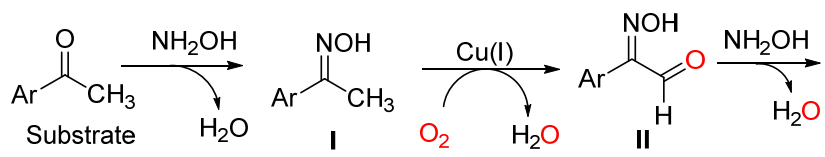

III

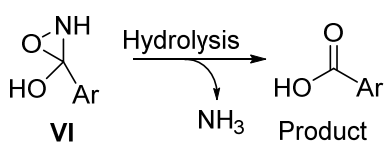

图式 $21 \mathrm{Cu}$ 催化由酮生成酸反应的可能机理

Scheme 21 Mechanisms proposed for $\mathrm{Cu}$-catalyzed synthesis of acids from ketones

$\mathrm{C}\left(\mathrm{sp}^{3}\right)$ 单键氧化断裂生成酮和羧酸(Eq. 17), 该反应中碱 $\mathrm{hppH}$ 发挥着重要作用而且不可取代.

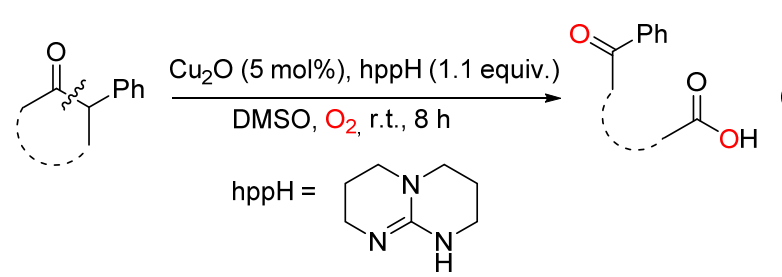

Schoenebeck等认为, 底物酮 $\mathbf{I}$ 通过互变生成烯醇式 II, II 与 $\mathrm{Cu}(\mathrm{II})$ 在氧气下形成 $\mathrm{Cu}(\mathrm{II})$ 过氧化合物 III，该化 合物通过两种可能路径生成碳一碳单键断裂产物，一种 是阴离子路径(Path A), 通过电子转移, 开环生成目标 化合物 $\mathbf{V}$; 另外一种是(Path B)通过自由基均裂, 再与氧 气作用生成 $\mathrm{C}\left(\mathrm{sp}^{2}\right)(\mathrm{CO})-\mathrm{C}\left(\mathrm{sp}^{3}\right)$ 单键断裂产物 $\mathbf{V}$ (Scheme 22).
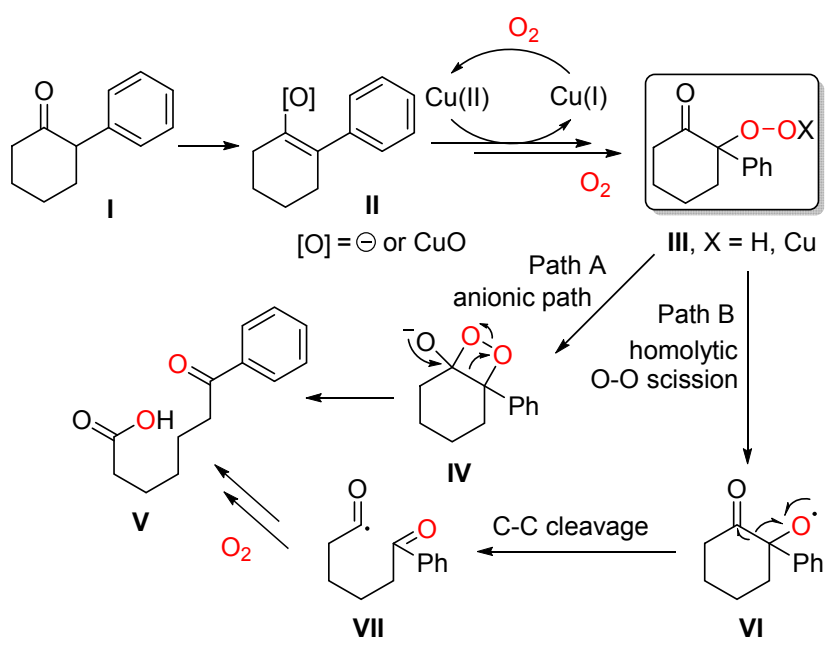

图式 $22 \mathrm{Cu}_{2} \mathrm{O}$ 催化环酮生成酸反应可能的反应机理

Scheme 22 Mechanisms proposed for $\mathrm{Cu}_{2} \mathrm{O}$ catalyzed synthesis of acids from cyclic ketones 
2015 年, Yin 课题组 ${ }^{[29]}$ 使用杂多酸在氧气下将 5-羟 甲基糠醛氧化成马来酸酐(Scheme 23), 他们认为该反 应分两步进行, 先进行 $\mathrm{C}\left(\mathrm{sp}^{3}\right)-\mathrm{C}\left(\mathrm{sp}^{3}\right)$ 单键氧化断裂成 5-羟基-2-呋喃甲醛, 然后再进一步氧化为目标产物. 他 们认为该反应是通过电子转移和氧转移(ET-OT)机理进 行，在该过程中杂多酸中 $\mathrm{V}$ 由五价还原为四价.

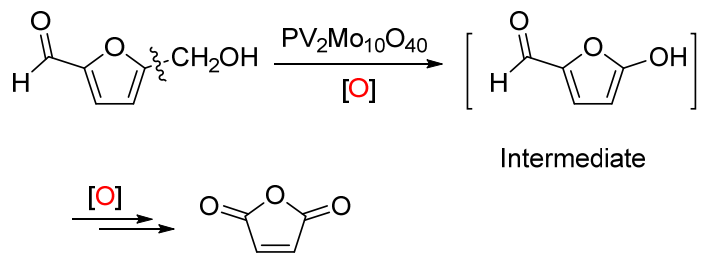

图式 23 杂多酸催化 $\mathrm{C}\left(\mathrm{sp}^{3}\right)-\mathrm{C}\left(\mathrm{sp}^{3}\right)$ 单键氧化断裂 Scheme 23 Heteropolyacid catalyzed oxidative cleavage of $\mathrm{C}\left(\mathrm{sp}^{3}\right)-\mathrm{C}\left(\mathrm{sp}^{3}\right)$ bond

\subsection{4 $\mathrm{C}\left(\mathrm{sp}^{2}\right)(\mathrm{CO})-\mathrm{C}\left(\mathrm{sp}^{3}\right)$ 单键断裂生成酯}

2013 年, Jiao 课题组 ${ }^{[30]}$ 以 $\mathrm{CuBr}$ 为催化剂, 在吡啶 和一个大气压氧气下, 催化 1,3-二酮化合物氧化酯化生 成 $\alpha$-酩酸酯的反应(Eq. 18). 该法高效地将 $\mathrm{C}\left(\mathrm{sp}^{2}\right)-$ $(\mathrm{CO})-\mathrm{C}\left(\mathrm{sp}^{3}\right)$ 单键氧化断裂再与醇反应生成 $\alpha$-酮酸酯.

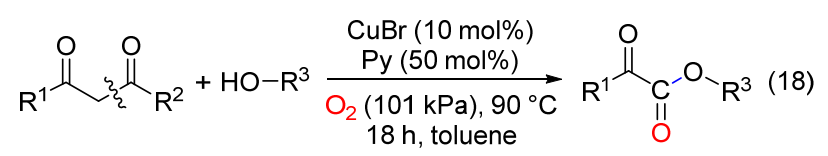

Jiao 等认为, 在 $\mathrm{Cu}$ 催化条件下, 底物 I 和 II 先经过 脱氢偶联生成中间体 IV, 中间体 IV 在 $\mathrm{Cu} / \mathrm{O}_{2}$ 催化下被 氧化为过氧化中间体 $\mathbf{V}, \mathbf{V}$ 通过单电子转移得到阴离子 中间体 VI 和羟基自由基, VI 和 II 反应生成半缩醛中间 体 VII, 最后再通过氧化使 $\mathrm{C}\left(\mathrm{sp}^{2}\right)(\mathrm{CO})-\mathrm{C}\left(\mathrm{sp}^{3}\right)$ 单键裂解 得到目标化合物 III 和副产物 VIII (Scheme 24).

2014 年, Jiao 课题组 ${ }^{[31]}$ 又报道了芳香酮或脂肪酮 在空气中被 $\mathrm{CuBr}-\mathrm{Py}$ 催化发生 $\mathrm{C}\left(\mathrm{sp}^{2}\right)(\mathrm{CO})-\mathrm{C}\left(\mathrm{sp}^{3}\right)$ 单键 氧化断裂并与醇酯反应生成酯(Eq. 19), 他们使用该方 法合成了 65 个最高收率达 $90 \%$ 的目标产物. 该反应适 用于多种不同种类的酮, 甚至长脂肪链的酮, 醇的种类 也可以有多种, 包括伯醇、仲醇等, 并且含有手性的醇 构型能得到保持.

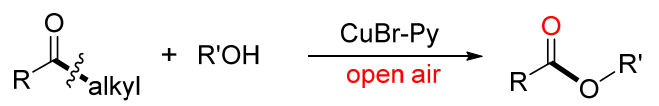

$$
\begin{aligned}
& \mathrm{R}=\mathrm{Ar} \text {, alkenyl }
\end{aligned}
$$

Jiao 等认为首先酮 $\mathbf{I}$ 与醇发生可逆的亲核加成反应 得到半缩酮 II , 然后反应通过两种可能路径进行 (Scheme 25). Path A: 半缩酮 II 发生单电子转移与 $\mathrm{Cu}(\mathrm{II})$ 生成以碳为中心的自由基中间体, 该中间体与分

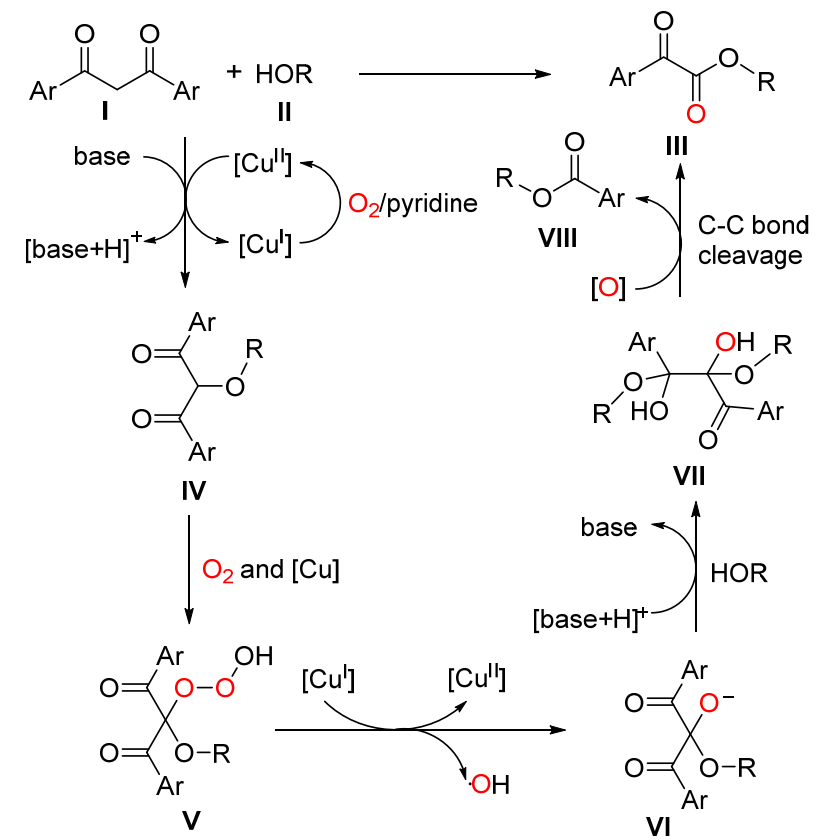

图式 $24 \mathrm{CuBr}$ 催化 1,3-二酮合成 $\alpha$-酮酸酯反应的可能机理 Scheme 24 Mechanisms proposed for $\mathrm{CuBr}$ catalyzed synthesis of $\alpha$-ketoesters from 1,3-diones

子氧生成过氧自由基 III, III 质子化后生成 IV, IV 最后 通过电子化转移使碳一碳单键断裂生成目标化合物 $\mathbf{V}$, 同时脱去一分子醛; Path B: 半缩酮 II 脱水生成乙烯醚 VI, VI 与 $\mathrm{Cu}(\mathrm{II})$ 和超氧游离基反应得到 1,2-二氧杂环丁 烷中间体 VII, VII 最后通过碳一碳单键和氧-氧键的断裂 生成目标化合物 VIII，同时脱去一分子醛.

2016 年, $\mathrm{He}$ 课题组 ${ }^{[32]}$ 报道了类似的反应(Eq. 20), 该反应使用 $\mathrm{CuCl}_{2} \cdot \mathrm{H}_{2} \mathrm{O}$ 作为催化剂, 在氧气下进行, 不需要吡啶参与，条件相对温和，对多种官能团耐受. 该反应中使用 $\mathrm{LiBr}$, 将 $\mathrm{Cu}(\mathrm{II})$ 还原为 $\mathrm{Cu}(\mathrm{I}), \mathrm{Cu}(\mathrm{I})$ 在氧气 下生成 $\mathrm{Cu}(\mathrm{II})$, 实现催化剂的循环.

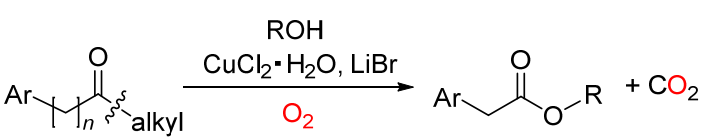

$\mathrm{He}$ 等提出了不同的反应机理，他们认为底物芳基 乙酮 I 通过三次连续的催化氧化, 形成丙酮酸结构的中 间体 IV，IV 再与醇发生酯化反应和亲核加成反应得到 中间体 VI, VI 最后脱去一分子甲酸乙酯生成目标产物 VII (Scheme 26).

\subsection{5 $\mathrm{C}\left(\mathrm{sp}^{2}\right)(\mathrm{CO})-\mathrm{C}\left(\mathrm{sp}^{3}\right)$ 单键断裂生成酰胺}

2014 年, Kaliappan 课题组 ${ }^{[33]}$ 使用 $\mathrm{CuCl}_{2}$ 催化酮与 $N$-杂环芳伯胺一锅法合成了酰胺(Eq. 21), 该反应条件 温和、对酸碱敏感的底物同样适用. 


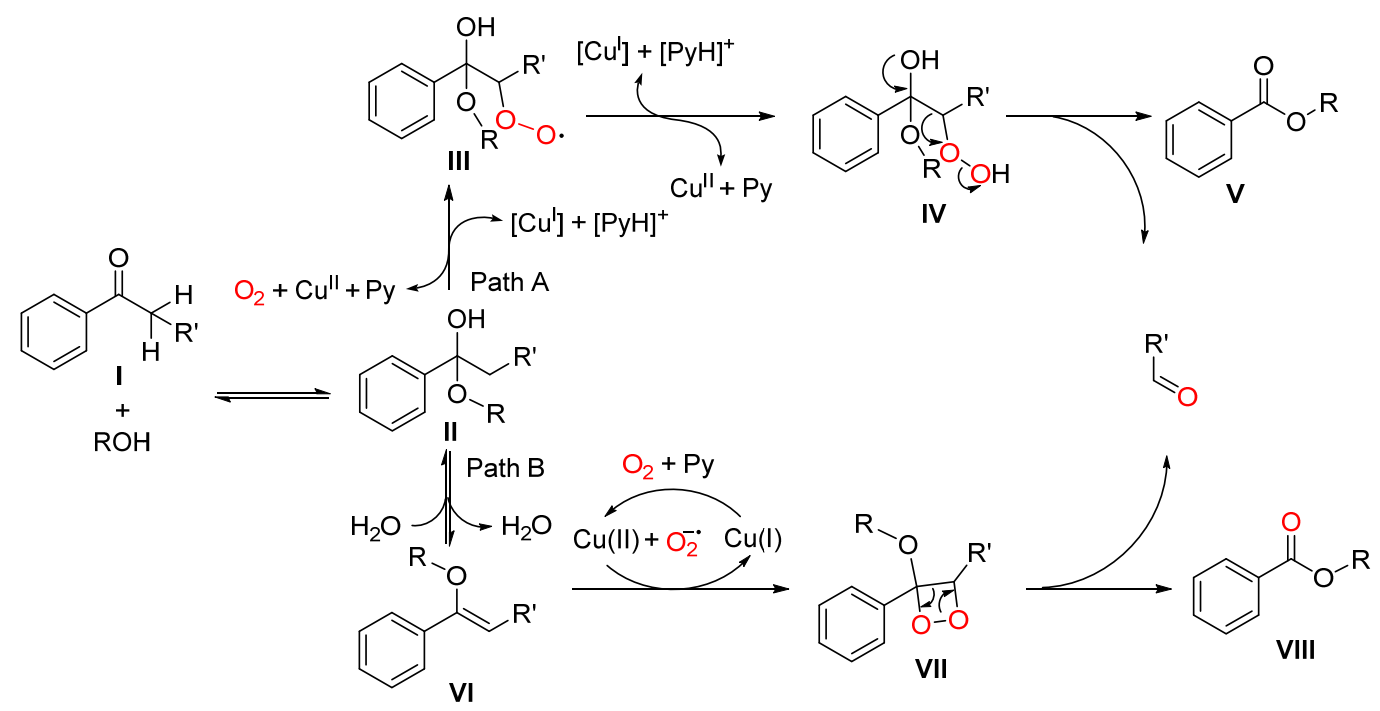

图式 $25 \mathrm{CuBr}-\mathrm{Py}$ 催化酮合成酯反应的可能机理

Scheme 25 Mechanisms proposed for CuBr-Py catalyzed synthesis of esters from ketones

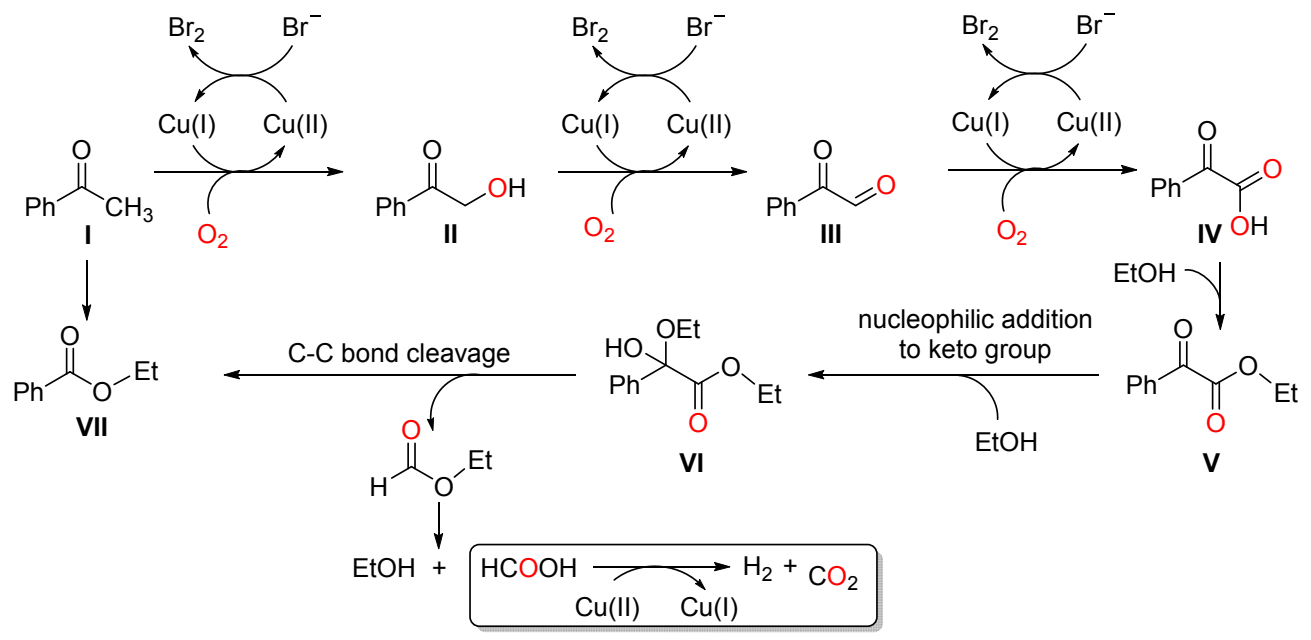

图式 $26 \mathrm{CuCl}_{2}$ 催化由酮合成酯反应可能机理

Scheme 26 Mechanisms proposed for $\mathrm{CuCl}_{2}$ catalyzed synthesis of esters from ketones

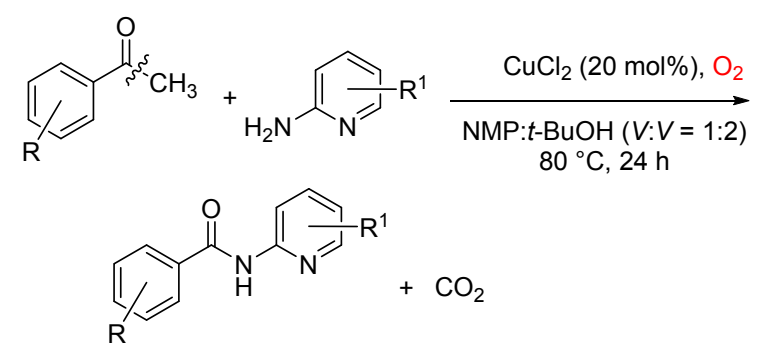

Kaliappan 等认为该反应可能经由酮和 2-氨基吡啶 先进行可逆的缩合反应生成烯胺 $\mathbf{I}, \mathbf{I}$ 在 $\mathrm{Cu}(\mathrm{I})$ 催化下氧 化生成过氧自由基 II, II 与双键加成生成 1,2-二氧杂环 丁烷类中间体 III, III 开环在碱和 $\mathrm{Cu}(\mathrm{II}) \mathrm{OH} / \mathrm{O}_{2}$ 作用下分 解为不稳定酮醛和 2-氨基吡啶, 接着脱水缩合生成咪唑 并吡啶酮 $\mathbf{V}, \mathbf{V}$ 通过互变生成 $\mathbf{V I}$ 后, 在 $\mathrm{Cu}(\mathrm{I}) / \mathrm{O}_{2}$ 催化氧
化下生成过氧化自由基 VII, 然后与双键生成 1,2-二氧 杂环丁烷类中间体 VIII，最后通过开环并脱羧生成目标 化合物 X (Scheme 27).

2014 年, Jiao 课题组 ${ }^{[34]}$ 报道了在氧气下, $\mathrm{CuCl}_{2}$ 催化 的芳酮与叠氮化钠反应生成酰胺的反应(Eq. 22). 该反 应不仅可以用于芳乙酮同时也适用于长链的芳香酮的 $\mathrm{C}\left(\mathrm{sp}^{2}\right)(\mathrm{CO})-\mathrm{C}\left(\mathrm{sp}^{3}\right)$ 单键氧化断裂, 提供了一种新的合 成酰胺的方法.

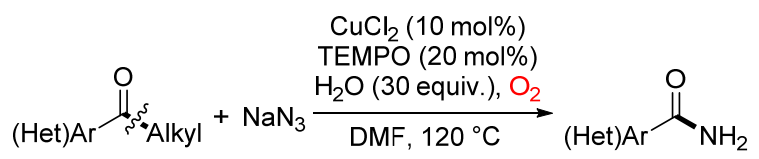

Jiao 等认为底物酮 $\mathbf{I}$ 先与叠氮化钠进行可逆亲核加 


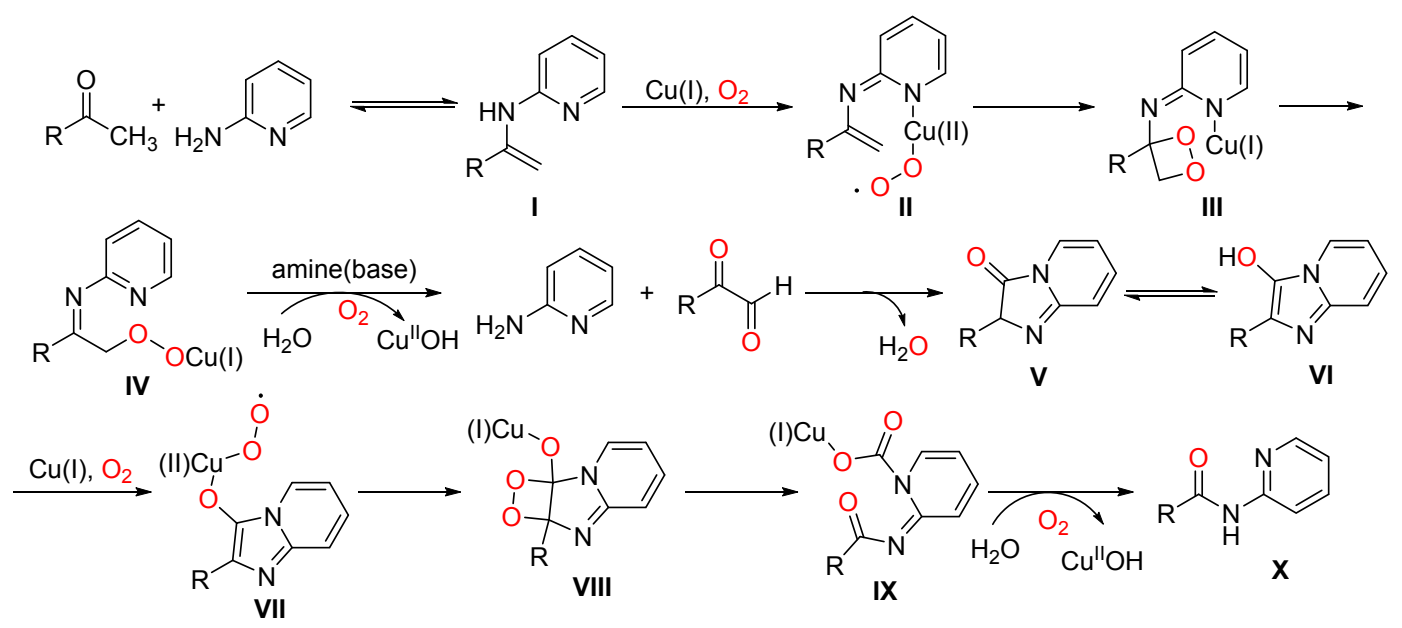

图式 $27 \mathrm{CuCl}_{2}$ 催化甲基酮合成 $N$-杂环酰胺反应的可能机理

Scheme 27 Mechanisms proposed for $\mathrm{CuCl}_{2}$ catalyzed synthesis of $\mathrm{N}$-heterocyclic amides from methyl ketones

成反应得到不稳定的中间体 II, 该中间体在 $\mathrm{Cu} / \mathrm{O}_{2}$ 催化 系统下被氧化羟基化得到中间体 III, 中间体 III 的共振 式 IV 通过电子转移使 $\mathrm{C}\left(\mathrm{sp}^{2}\right)(\mathrm{CO})-\mathrm{C}\left(\mathrm{sp}^{3}\right)$ 单键断裂生成 $\mathbf{V}$, 同时释放一分子醛和氮气, $\mathbf{V}$ 共振即得目标化合物 VI (Scheme 28).

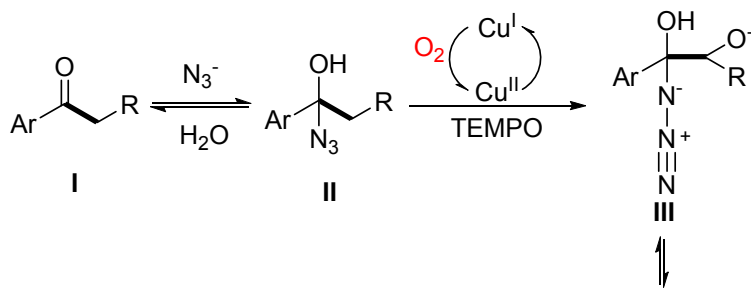

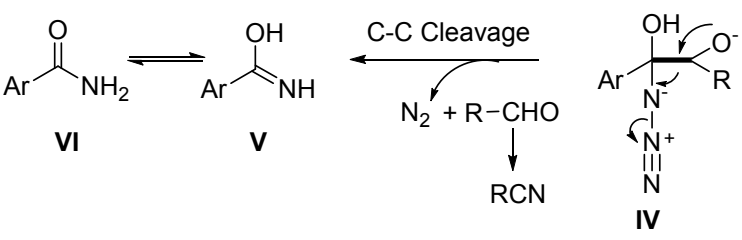

图式 $28 \mathrm{CuCl}_{2}$ 催化酮合成酰胺反应的可能机理

Scheme 28 Mechanisms proposed for $\mathrm{CuCl}_{2}$ catalyzed synthesis of amides from ketones

2015 年, Song 课题组 ${ }^{[35]}$ 使用 $\mathrm{CuBr}$ 作为催化剂, 在 吡啶和氧气下, 催化酮的 $\mathrm{C}\left(\mathrm{sp}^{2}\right)(\mathrm{CO})-\mathrm{C}\left(\mathrm{sp}^{3}\right)$ 单键断裂 与唑类芳杂环反应生成三级酰胺(Eq. 23).

$$
\text { (Het)Ar } \stackrel{\mathrm{CuBr}(10 \mathrm{~mol} \%)}{\stackrel{\mathrm{Cu}}{\text { pyridine }(3.0 \text { equiv. })}}
$$

Song 等认为该反应通过两种可能路线进行(Scheme
29). Path $\mathrm{A}$ : 在 $\mathrm{Cu}(\mathrm{II})$ 和氧气下, 底物 $\mathbf{I}$ 中的亚甲基首先 通过单电子转移被氧化为过氧化自由基中间体 II, 然后 被芳杂环亲核进攻生成中间体 III, III 质子化生成中间 体 IV, IV 通过电子转移, 使 $\mathrm{C}\left(\mathrm{sp}^{2}\right)(\mathrm{CO})-\mathrm{C}\left(\mathrm{sp}^{3}\right)$ 单键发 生断裂生成目标化合物 $\mathbf{V}$, 同时释放出一分子醛和水. Path B: I 先与芳杂环发生亲核加成反应生成中间体 VI, 然后脱去一分子水得到中间体 VII, VII 再在 $\mathrm{Cu}(\mathrm{II}) / \mathrm{O}_{2}$ 催化氧化系统作用下生成 1,2-二氧杂环丁烷中间体 VIII, VIII 通过 $\mathrm{C}\left(\mathrm{sp}^{2}\right)(\mathrm{CO})-\mathrm{C}\left(\mathrm{sp}^{3}\right)$ 单键和氧一氧键发生 断裂生成目标化合物 $\mathbf{V}$, 同时释放出一分子醛和水.

2014 年, $\mathrm{Yin}$ 课题组 ${ }^{[36]}$ 报道了氧气下, $\mathrm{CuCl}$ 催化不 同分子的 $\mathrm{C}\left(\mathrm{sp}^{2}\right)(\mathrm{CO})-\mathrm{C}\left(\mathrm{sp}^{3}\right)$ 单键和碳 - 氮单键断裂一锅 法生成叔酰胺的方法(Eq. 24), 该反应不需要碱和配体.

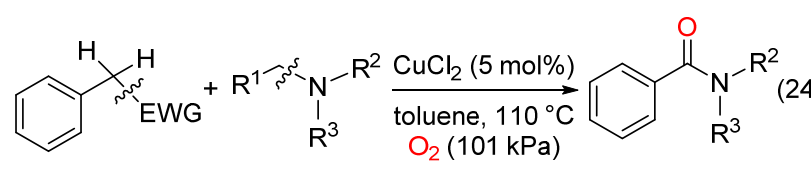

$\mathrm{EWG}=\mathrm{CHO}, \mathrm{COCH}_{2} \mathrm{Ph}, \mathrm{COOH}$

2012 年, Jiao 课题组 ${ }^{[37]}$ 在氧气下, 使用 $\mathrm{Mn}(\mathrm{OAc})_{3}$ 催化醛的 $\mathrm{C}\left(\mathrm{sp}^{2}\right)(\mathrm{CO})-\mathrm{C}\left(\mathrm{sp}^{3}\right)$ 单键断裂与胺生成了甲酰 胺(Eq. 25), 他们认为该反应是一个自由基反应，并使用 该方法合成了 27 个收率最高可达 $96 \%$ 的目标化合物.

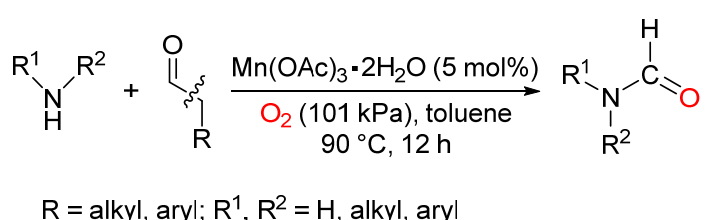

Jiao 等认为胺 I 先与醛 II 进行脱水反应生成亚胺 III, 在 $\mathrm{Mn}(\mathrm{OAc})_{3}$ 催化下 III 被氧化生成过氧游离基 IV, 


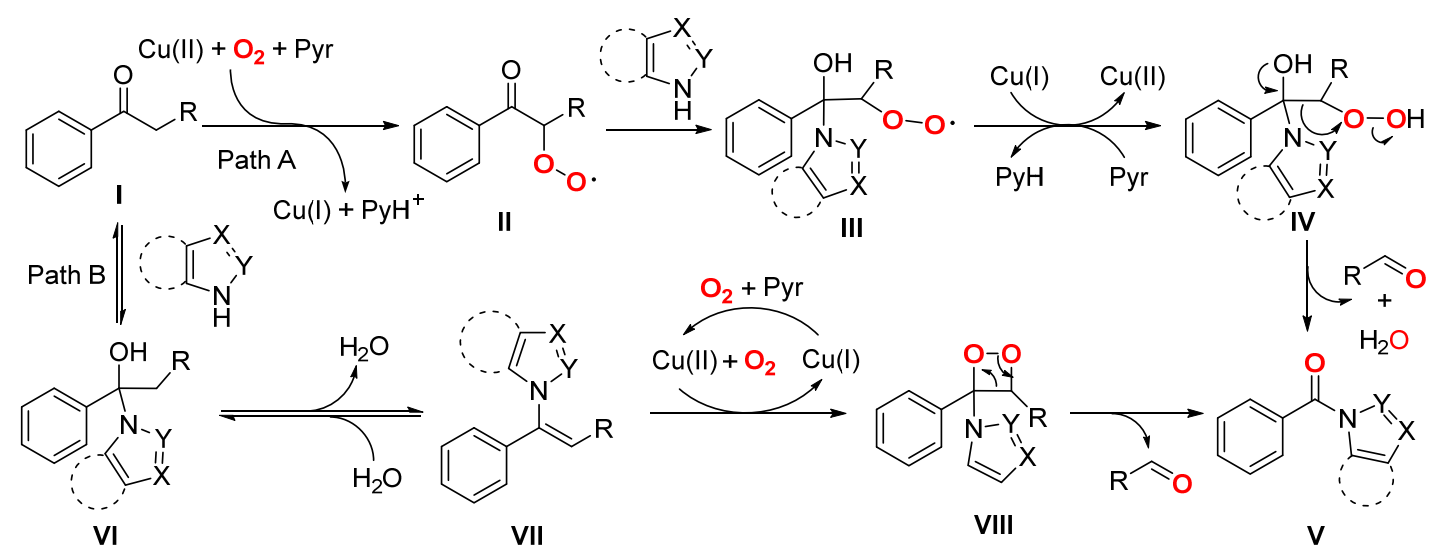

图式 $29 \mathrm{CuBr}$ 催化由芳香乙酮合成三级酰胺反应的可能机理

Scheme 29 Mechanisms proposed for $\mathrm{CuBr}$ catalyzed synthesis of tertiary amides from ketones

接着进行分子内加成生成 1,2-二氧杂环丁烷自由基 $\mathbf{V}, \mathbf{V}$ 在氧气下可以与亚胺 III 反应生成中间体 VI 并释放 IV, 使得反应继续发生. 最后 VI 发生裂解反应得到目标化 合物 VII 以及醛和一些未知的聚合物(Scheme 30).

2014年, Xu 课题组 ${ }^{[38]}$ 报道了 CuI 催化的邻位溴代的 芳基乙酰胺, 通过断裂 $\mathrm{C}\left(\mathrm{sp}^{2}\right)(\mathrm{CO})-\mathrm{C}\left(\mathrm{sp}^{3}\right)$ 单键和碳-氮 单键脱去一分子一氧化碳, 然后关环生成环喹唑啉酮 (Eq. 26).

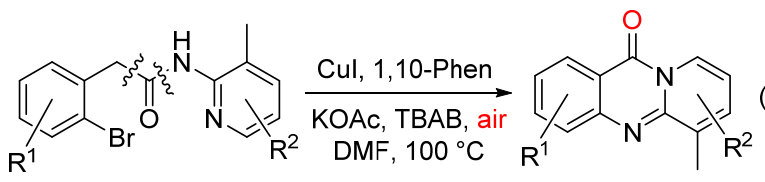

$\mathrm{Xu}$ 等认为该反应经由 Scheme 31 的机理进行: 首 先底物 I 被氧化生成 $\alpha$-酮酰胺 II, 该化合物再在 $\mathrm{Cu}(\mathrm{I})$ 的催化下生成中间体 III, III 脱去一分子卤化氢后发生 分子内反应生成中间体 IV, 然后吡啶环上的氮原子亲 核进攻酮崖基得到一个两性离子中间体 VI, VI 通过电 子转移脱去一分子一氧化碳得到目标化合物 VII.

\subsection{6 $\mathrm{C}\left(\mathrm{sp}^{2}\right)(\mathrm{CO})$ - $\mathrm{C}\left(\mathrm{sp}^{3}\right)$ 单键断裂生成杂环}

2016 年, Yan 和 Liu 课题组 ${ }^{[39]}$ 报道了一种氧气下 $\mathrm{Cu}(\mathrm{OAc})_{2}$ 催化芳乙酸氧化脱羧并与邻氨基芳酮反应高 效地生成杂环 2-芳基喹唑啉方法(Eq. 27), 该方法通过 一步生成多个碳-氮键.

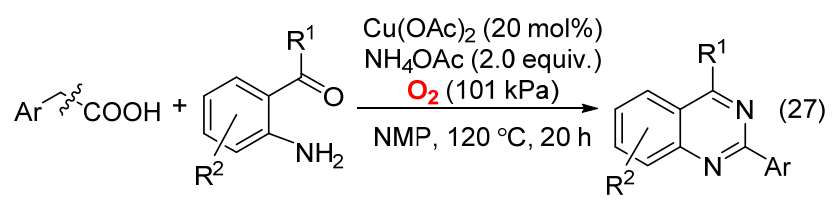

Liu 等认为底物 I 先与 $\mathrm{Cu}(\mathrm{II})$ 反应通过配体交换生 成 $\mathrm{Cu}$ 络合物 II, II 通过脱去一分二氧化碳生成 III, 氧 气插入到 III 生成中间体 IV, IV 转化为 $(\mathrm{HO}) \mathrm{Cu}(\mathrm{OAc})$ 并 通过 $\beta-\mathrm{H}$ 消除生成苯甲醛, ( $\mathrm{HO}) \mathrm{Cu}(\mathrm{OAc})$ 在醋酸作用下 重新生成 $\mathrm{Cu}(\mathrm{OAc})_{2}$, 苯甲醛与 $\mathbf{V}$ 缩合生成 $\mathbf{V I}, \mathbf{V I}$ 通过 温和的分子内亲核加成和 $\mathrm{Cu}$ 催化的氧气氧化得到目标 化合物 VIII (Scheme 32).

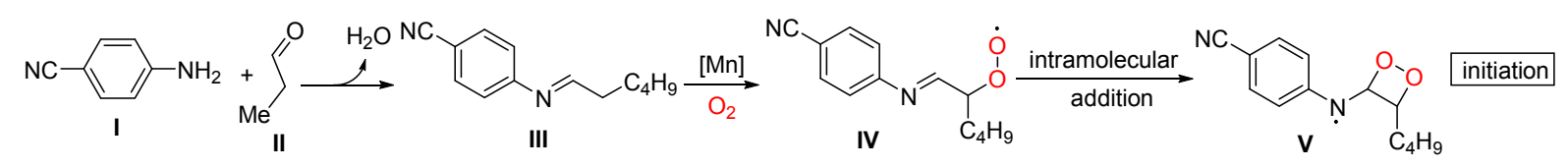

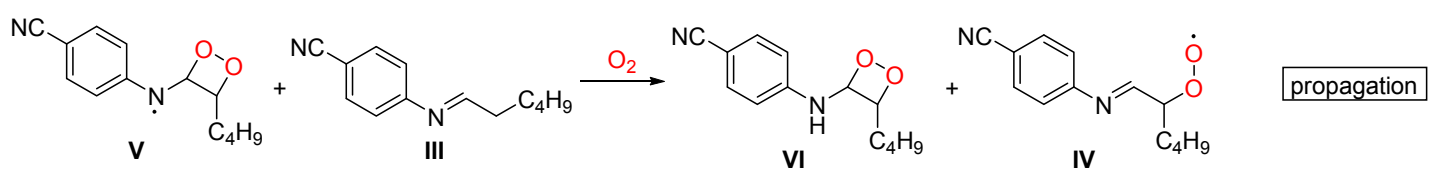

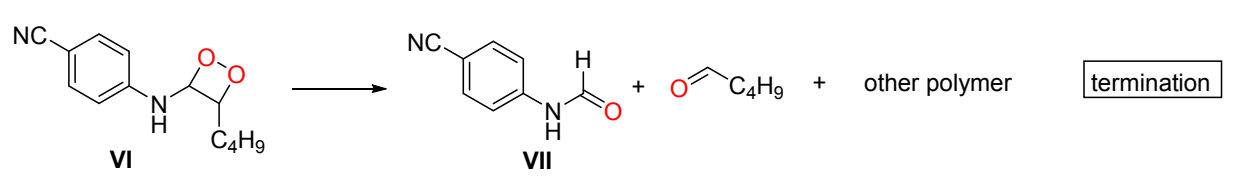

图式 $30 \mathrm{Mn}$ 催化甲酰胺合成反应的可能机理

Scheme 30 Mechanisms proposed for Mn-catalyzed synthesis of formamides 


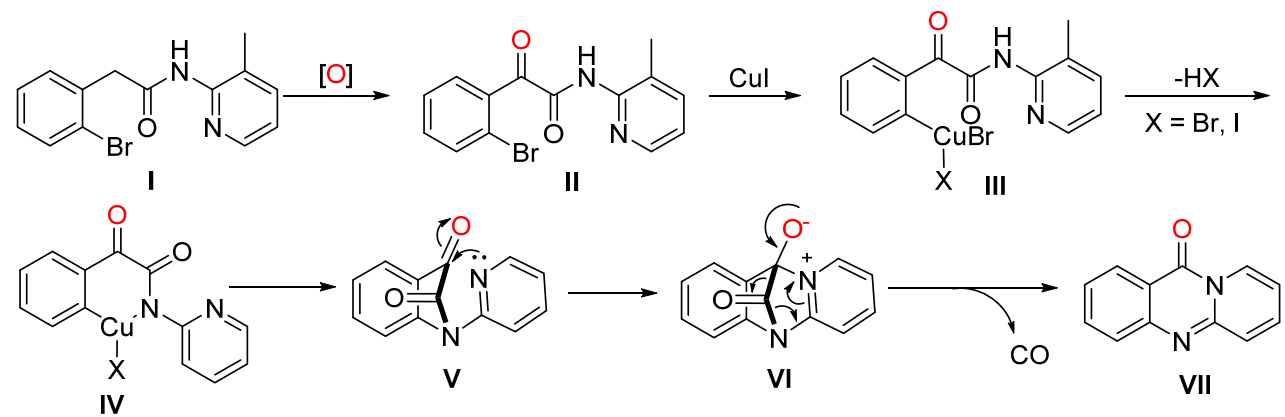

图式 $31 \mathrm{CuI}$ 催化芳基乙酰胺合成喹唑酮反应的可能机理

Scheme 31 Mechanisms proposed for $\mathrm{CuI}$ catalyzed synthesis of quinazolinones from arylacetamides

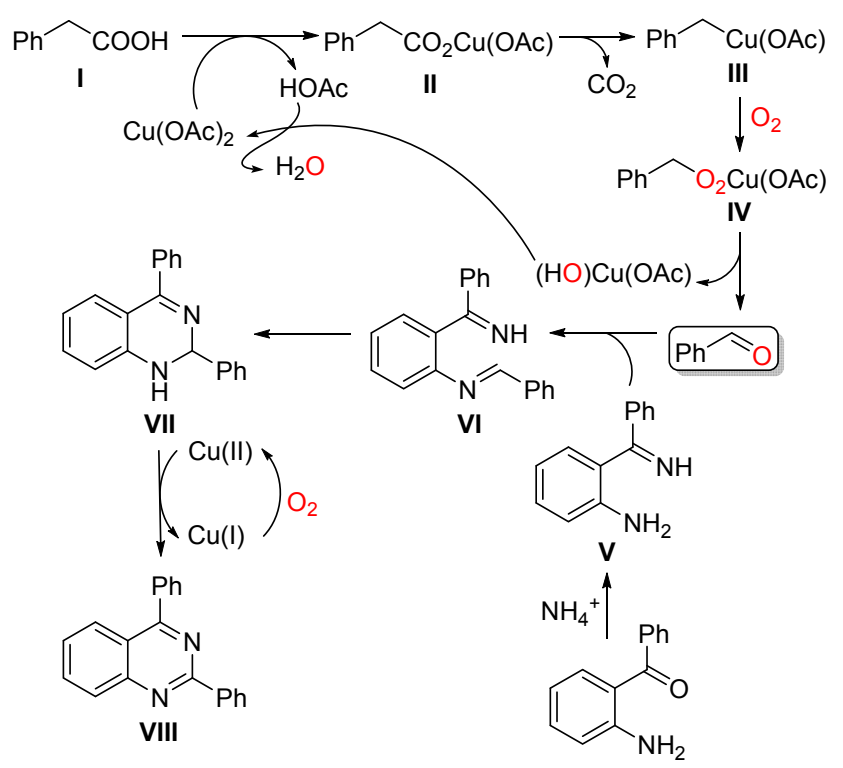

图式 $32 \mathrm{Cu}(\mathrm{OAc})_{2}$ 催化 2-芳基喹唑啉合成反应的可能机理 Scheme 32 Mechanisms proposed for $\mathrm{Cu}(\mathrm{OAc})_{2}$ catalyzed synthesis 2-arylquinazolines

$1.3 \mathrm{C}\left(\mathrm{sp}^{2}\right)(\mathrm{CO})-\mathrm{C}\left(\mathrm{sp}^{2}\right)$ 和 $\mathrm{C}\left(\mathrm{sp}^{2}\right)(\mathrm{CO})-\mathrm{C}(\mathrm{sp})$ 单键断 裂的反应

2016 年, Monguchi 和 Sajiki 课题组 ${ }^{[40]}$ 首次报道了在 氧气下 $\mathrm{Pd} / \mathrm{C}$ 催化肉桂酫或苯丙醛类化合物(前者为 $\mathrm{C}\left(\mathrm{sp}^{2}\right)-\mathrm{C}\left(\mathrm{sp}^{3}\right)$ 后者为 $\left.\mathrm{C}\left(\mathrm{sp}^{2}\right)-\mathrm{C}\left(\mathrm{sp}^{2}\right)\right)$ 生成苯乙烯类化合 物(Eq. 28).

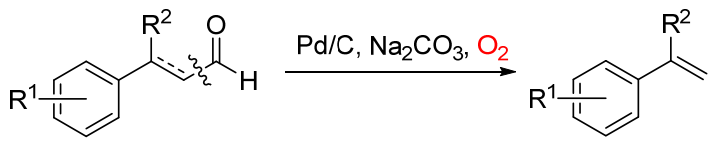

Sajiki 等认为 $\operatorname{Pd}(0) / C$ 先插入到醛 I 醛基的碳氢之间, 得到酰基 $\mathrm{Pd}(\mathrm{II}) / \mathrm{H}$ 复合物 II, 接着 $\mathrm{Pd}$ 发生迁移生成乙烯 基 $\mathrm{Pd}(\mathrm{II}) / \mathrm{H}$ 复合物 III, III 通过还原消除得到苯乙烯类化 合物并同时生成一氧化碳和 $\operatorname{Pd}(0) / C$ (Scheme 33).

2016 年, Song 课题组 ${ }^{[41]}$ 报道了氧气下, $\mathrm{Cu} / \mathrm{Fe}$ 混合 盐催化剂催化 $\alpha, \beta$-不饱和羰基化合物的 $\mathrm{C}\left(\mathrm{sp}^{2}\right)(\mathrm{CO})$ $\mathrm{C}\left(\mathrm{sp}^{2}\right)$ 单键断裂, 并与氢亚磷酸二酯反应生成 $\beta$-酮磷酸 酯(Eq. 29).

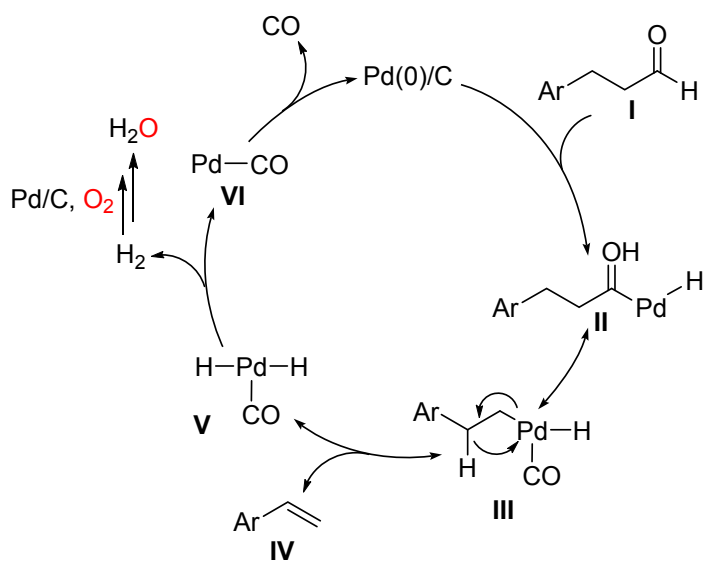

图式 $33 \mathrm{Pd}$ 催化的 $\mathrm{C}\left(\mathrm{sp}^{2}\right)-\mathrm{C}\left(\mathrm{sp}^{3}\right)$ 单键断裂反应的可能机理 Scheme 33 Mechanisms proposed for Pd-catalyzed cleavage of the $\mathrm{C}\left(\mathrm{sp}^{2}\right)-\mathrm{C}\left(\mathrm{sp}^{3}\right)$ bond

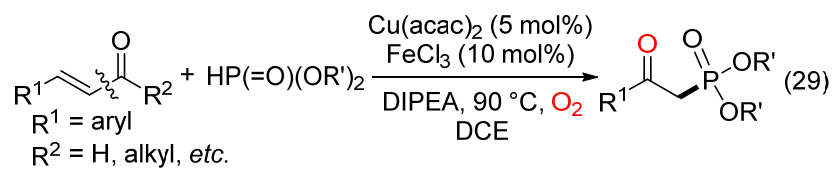<smiles>O=C(C=Cc1ccccc1)C=Cc1ccccc1</smiles>

$85 \%$<smiles>CC(=O)C=Cc1ccccc1</smiles>

$62 \%$<smiles>O=CC=Cc1ccccc1</smiles>

$67 \%$
2015 年, Song 课题组 ${ }^{[42]}$ 报道了苯炔酸在氧气和廉 价的 $\mathrm{Cu} / \mathrm{Fe}$ 催化系统下, 通过 $\mathrm{C}\left(\mathrm{sp}^{2}\right)(\mathrm{CO})-\mathrm{C}(\mathrm{sp})$ 单键断 裂脱羧后与氢亚磷酸二酯反应生成 $\beta$-酮磷酸酯类化合 物的反应(Eq. 30), 并通过该法得到 19 个收率在 33\% $78 \%$ 目标化合物.

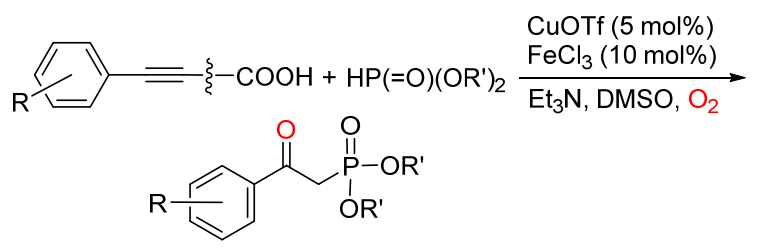

Song 等认为, $\mathbf{I}$ 在 $\mathrm{Fe}(\mathrm{III})$ 和氧气作用下生成阳离子 基 II, II 在三乙胺作用下脱质子生成磷酰基自由基 III, 其与炔烃反应生成自由基 IV, IV 过氧化自由基 $\mathrm{V}$ 反应 
得到过氧化物 VI, VI 氢亚磷酸二酯作用下转化为目标 化合物 VIII (Scheme 34).

\section{2 氧气参与的非金属催化的无张力碳一碳单键 断裂}

金属催化的碳一碳单键断裂反应往往能取得较好的 效果, 但有些金属(如 Pd 等)价格昂贵, 回收成本高, 所 以近年来非金属(或无金属)催化的无张力碳-碳单键断 裂反应也引起了人们的关注.

2012 年, Jiang 课题组 ${ }^{[43]}$ 报道了氧气参与的非金属 催化的 $\alpha$-羟基芳基酮发生 $\mathrm{C}\left(\mathrm{sp}^{2}\right)(\mathrm{CO})-\mathrm{C}\left(\mathrm{sp}^{3}\right)$ 单键断裂
并与芐澳发生酯化的反应(Eq. 31).

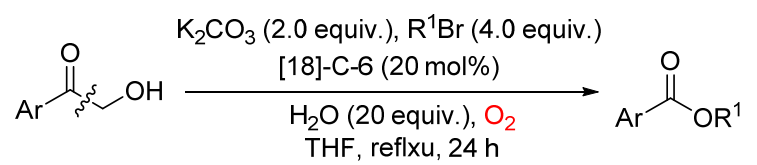

Jiang 等认为(Path A)底物 I 在碱、溴化苄和氧气下 通过自由基中间体生成二聚体 II, II 在碱和氧气下生成 双过氧化自由基 III, III 在 $\mathrm{BnBr}$ 作用下生成 1,2-二氧杂 环丁烷结构的中间体 IV, IV 通过电子重排断裂, 释放一 分子甲酸和二氧化碳并生成目标化合物 $\mathbf{V}$ (Scheme 35).

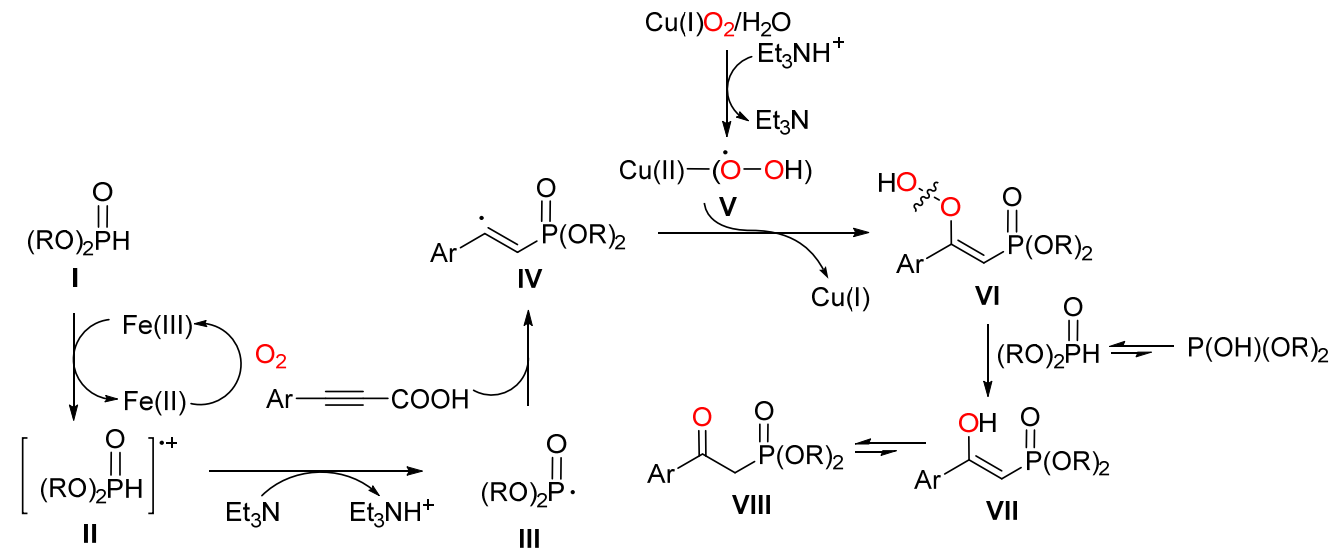

图式 $34 \mathrm{Cu} / \mathrm{Fe}$ 催化炔酸生成 $\beta$-酮磷酸酯反应的可能机理

Scheme 34 Mechanisms proposed for $\mathrm{Cu} / \mathrm{Fe}$-catalyzed synthesis of $\beta$-ketophosphonate from alkynyl carboxylic acids

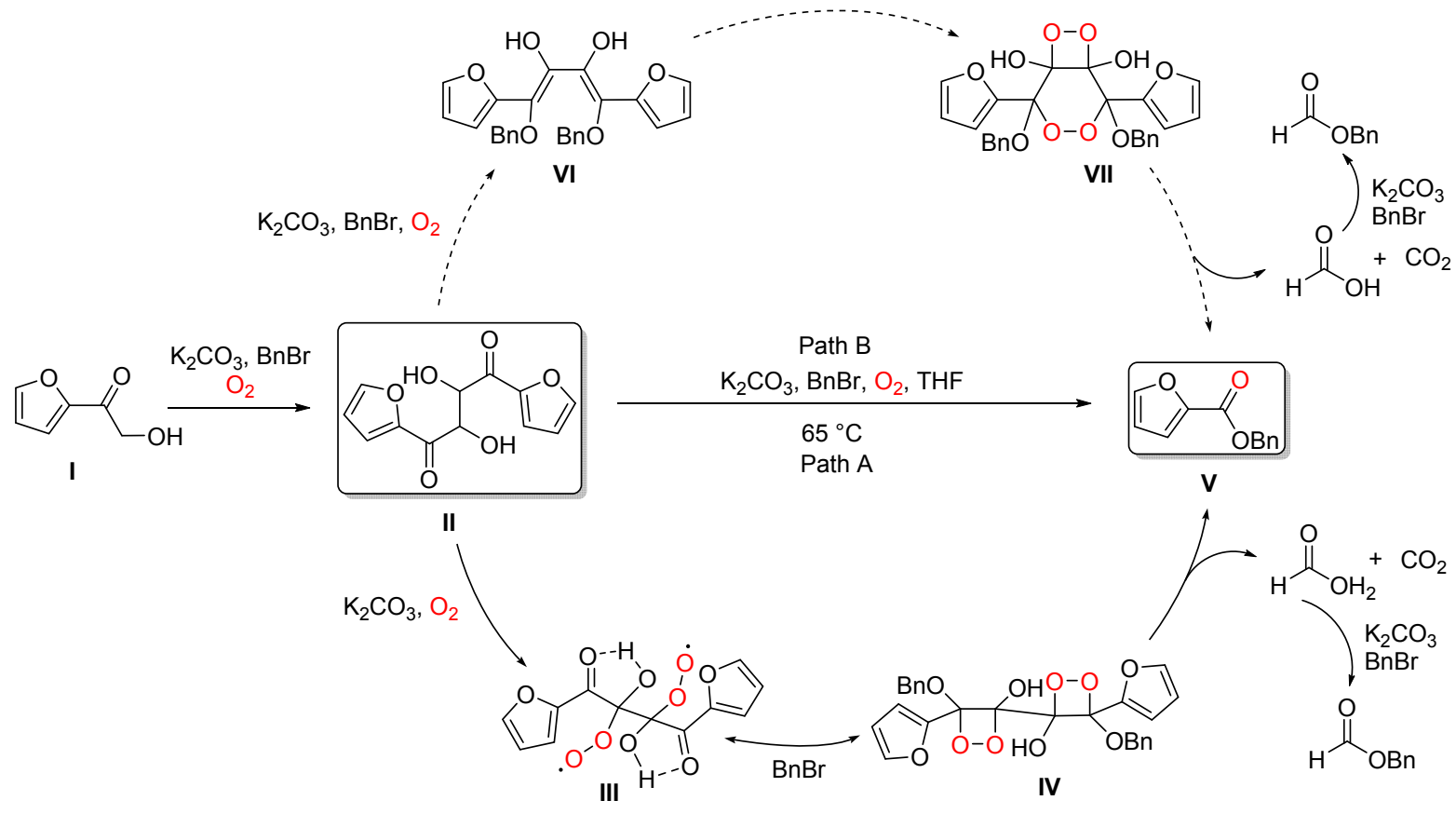

图式 35 非金属催化 $\mathrm{C}\left(\mathrm{sp}^{2}\right)(\mathrm{CO})-\mathrm{C}\left(\mathrm{sp}^{3}\right)$ 单键氧化断裂反应的可能机理

Scheme 35 Mechanisms proposed for transition-metal-free aerobic oxidative cleavage of $\mathrm{C}\left(\mathrm{sp}^{2}\right)(\mathrm{CO})-\mathrm{C}\left(\mathrm{sp}^{3}\right)$ bond 
2013 年, Jiang 等 ${ }^{[44]}$ 又提出了另外一条路径(Path B), 二聚体 II 与 $\mathrm{K}_{2} \mathrm{CO}_{3}, \mathrm{BnBr}$ 在氧气下生成中间体 VI, VI 通过[4+2]和[2+2]加成得到 VII, VII 最后通过断裂 $\mathrm{C}\left(\mathrm{sp}^{2}\right)(\mathrm{CO})-\mathrm{C}\left(\mathrm{sp}^{3}\right)$ 单键成为目标产物 $\mathbf{V}$ (Scheme 35).

2015 年, $\mathrm{Chu}$ 课题组 ${ }^{[45]}$ 使用便宜的磺化硅胶作为催 化剂, 在空气中催化苯胺类化合物与 1,3-二酮类化合物 反应, 通过 $\mathrm{C}\left(\mathrm{sp}^{2}\right)(\mathrm{CO})-\mathrm{C}\left(\mathrm{sp}^{3}\right)$ 单键氧化断裂生成酰胺 (Eq. 32).

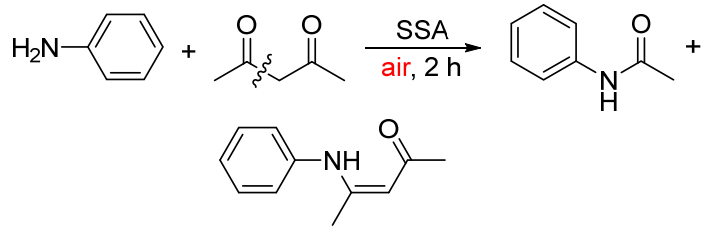

2016 年, Adimurthy 课题组 ${ }^{[46]}$ 报道了在氧气下, $\mathrm{AIBN}$ 促进 1,3-二酮 $\mathrm{C}\left(\mathrm{sp}^{2}\right)(\mathrm{CO})-\mathrm{C}\left(\mathrm{sp}^{3}\right)$ 单键断裂并与苯 胺反应生成酰胺(Eq. 33). 该反应是一个自由基反应.

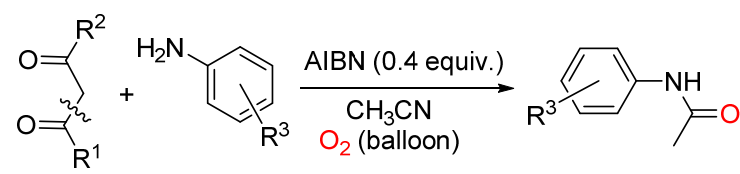

Adimurthy 等认为偶氮二异丁腈(AIBN)在加热条 件下通过失去氮气生成自由基 $\mathbf{I}, \mathbf{I}$ 在氧气下生成过氧化 自由基 II, 1,3-二酩与苯胺缩合生成的中间体 III 与过氧 化自由基 II 反应生成中间体 $\mathrm{V}, \mathrm{V}$ 通过电子转移形成不 稳定的 1,2-二氧杂环丁烷结构并通过断裂 $\mathrm{C}\left(\mathrm{sp}^{2}\right)(\mathrm{CO})-$ $\mathrm{C}\left(\mathrm{sp}^{3}\right)$ 单键生成目标化合物(Scheme 36).
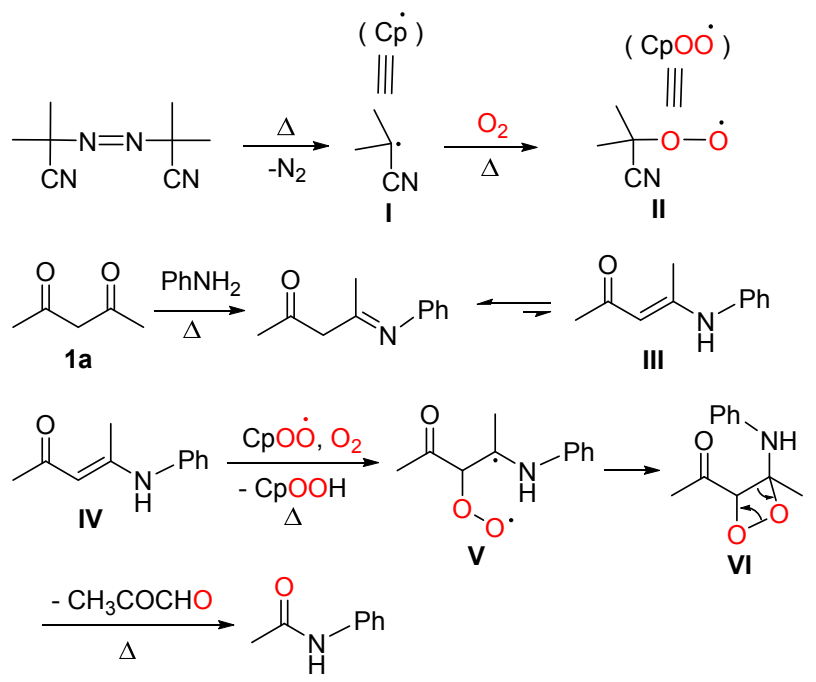

图式 $36 \mathrm{AIBN}$ 促进苯胺酰胺化反应的可能机理

Scheme 36 Mechanisms proposed for AIBN-promoted amidation of anilines

\section{3 结论与展望}

综上所述，氧气参与无张力碳一碳单键的断裂反应 近年来取得了较大的进展，但该类方法仍有一定的局限 性，比如还是以金属催化为主，并且需与羟基、羰基等 基团相连接. 氧气在该类反应中主要作为氧化剂氧化催 化金属或使底物生成不稳定的过氧化物而发挥作用. 通 过对该类反应的综述，希望对氧气在该类反应中的作用 机理有更加深刻的理解，相信在不久的将来，更加廉 价、环保、选择性高、并可持续的碳一碳单键断裂新方 法会不断被开发出来，这些新的方法最终会广泛应用于 有机合成、环境保护、工业生产等各个领域.

\section{References}

[1] (a) Padwa, A.; Zhang, H. J. Org. Chem. 2007, 72, 2570.

(b) Ikeda, S.; Shibuya, M.; Iwabuchi, Y. Chem. Commun. 2007, 38, 504.

[2] (a) Huber, G. W.; Iborra, S.; Corma, A. Chem. Rev. 2006, 106, 4044. (b) Hu, S.; Shima, T.; Hou, Z. Nature 2014, 512, 413.

[3] Seo, J.-H.; Lee, S.-M.; Lee, J.; Park, J.-B. J. Biotechnol. 2015, 216, 158.

[4] (a) Chen, F.; Wang, T.; Jiao, N. Chem. Rev. 2014, 114, 8613.

(b) Ruhland, K. Eur. J. Org. Chem. 2012, 2012, 2683.

(c) Jun, C. H. Chem. Soc. Rev. 2004, 33, 610.

(d) Liu, H.; Feng, M.; Jiang, X. Chem.-Asian J. 2014, 9, 3360.

(e) Dermenci, A.; Coe, J. W.; Dong, G. Org. Chem. Front. 2014, 1, 567.

(f) Amadio, E.; Di Lorenzo, R.; Zonta, C.; Licini, G. Coord. Chem. Rev. 2015, 301-302, 147.

(g) Murakami, M.; Ito, Y. Top. Organomet. Chem. 1999, 3, 97.

[5] Miyaura, N.; Suzuki, A.; Miyaura, N.; Suzuki, A. J. Chem. Soc., Chem. Commun. 1979, 19, 866.

[6] Heck, R. F. J. Am. Chem. Soc. 1968, 90, 5518.

[7] Sonogashira, K.; Tohda, Y.; Hagihara, N. Tetrahedron Lett. 1975, $16,4467$.

[8] Hanson, S. K.; Baker, R. T.; Gordon, J. C.; Scott, B. L.; Sutton, A. D.; Thorn, D. L. J. Am. Chem. Soc. 2009, 131, 428.

[9] Kirihara, M.; Yoshida, K.; Noguchi, T.; Naito, S.; Matsumoto, N.; Ema, Y.; Torii, M.; Ishizuka, Y.; Souta, I. Tetrahedron Lett. 2010, 51,3619 .

[10] Rozhko, E.; Raabova, K.; Macchia, F.; Malmusi, A.; Righi, P.; Accorinti, P.; Alini, S.; Babini, P.; Cerrato, G.; Manzoli, M.; Cavani, F. ChemCatChem 2013, 5, 1998.

[11] Liu, Z.-Q.; Zhao, L.; Shang, X.; Cui, Z. Org. Lett. 2012, 14, 3218.

[12] Zhao, Y.; Cai, S.; Li, J.; Wang, D. Z. Tetrahedron 2013, 69, 8129.

[13] Tnay, Y. L.; Chiba, S. Chem.-Asian J. 2015, 10, 873.

[14] Cooke, H. A.; Peck, S. C.; Evans, B. S.; van der Donk, W. A. J. Am. Chem. Soc. 2012, 134, 15660.

[15] Chen, Y. C.; Zhu, M. K.; Loh, T. P. Org. Lett. 2015, 17, 2712.

[16] Zhang, L; Bi, X. H.; Guan, X. X.; Li, Q.; Barry, B. D.; Liao, P. Q. Angew. Chem., Int. Ed. 2013, 52, 11303.

[17] Zhou, W.; Fan, W.; Jiang, Q.; Liang, Y.-F.; Jiao, N. Org. Lett. 2015, $17,2542$.

[18] Xing, Q.; Lv, H.; Xia, C.; Li, F. Chem. Commun. 2016, 52, 489.

[19] Wang, J.; Chen, W.; Zuo, S.; Liu, L.; Zhang, X.; Wang, J. Angew. Chem., Int. Ed. 2012, 51, 12334.

[20] Sun, H.; Yang, C.; Gao, F.; Li, Z.; Xia, W. Org. Lett. 2013, 15, 624. 
[21] Wang, Z.; Li, L.; Huang, Y. J. Am. Chem. Soc. 2014, 136, 12233.

[22] Zhang, C.; Wang, X.; Jiao, N. Synlett 2014, 45, 1458.

[23] Maji, A.; Rana, S.; Akanksha; Maiti, D. Angew. Chem., Int. Ed. 2014, 53, 2428.

[24] Yu, J.; Yang, H.; Jiang, Y.; Fu, H. Chem.-Eur. J. 2013, 19, 4271.

[25] Song, R. J.; Liu, Y.; Hu, R. X.; Liu, Y. Y.; Wu, J. C.; Yang, X. H.; Li, J. H. Adv. Synth. Catal. 2011, 353, 1467.

[26] Paria, S.; Halder, P.; Paine, T. K. Angew. Chem., Int. Ed. 2012, 51, 6299.

[27] Sathyanarayana, P.; Ravi, O.; Muktapuram, P. R.; Bathula, S. R. Org. Biomol. Chem. 2015, 13, 9681.

[28] Tsang, A. S. K.; Kapat, A.; Schoenebeck, F. J. Am. Chem. Soc. 2016, 138, 518.

[29] Lan, J.; Lin, J.; Chen, Z.; Yin, G. ACS Catal. 2015, 5, 2035.

[30] Zhang, C.; Feng, P.; Jiao, N. J. Am. Chem. Soc. 2013, 135, 15257.

[31] Xiaoqiang, H.; Xinyao, L.; Miancheng, Z.; Song, S.; Conghui, T.; Yizhi, Y.; Ning, J. J. Am. Chem. Soc. 2014, 136, 14858.

[32] Ma, R.; He, L.-N.; Liu, A.-H.; Song, Q.-W. Chem. Commun. 2016, $52,2145$.

[33] Parthasarathi, S.; Satrajit, I.; Kaliappan, K. P. Org. Lett. 2014, 16, 6212.

[34] Tang, C.; Jiao, N. Angew. Chem., Int. Ed. 2014, 53, 6528.
[35] Ding, W.; Song, Q. Org. Chem. Front. 2015, 2, 765.

[36] Chen, X.; Chen, T.; Li, Q.; Zhou, Y.; Han, L.-B.; Yin, S.-F. Chem. Eur. J. 2014, 20, 12234.

[37] Zhang, C.; Xu, Z.; Shen, T.; Wu, G.; Zhang, L.; Jiao, N. Org. Lett. 2012, 14, 2362.

[38] Sun, J.; Tan, Q.; Yang, W.; Liu, B.; Xu, B. Adv. Synth. Catal. 2014, 356, 388.

[39] Yan, Y.; Shi, M.; Niu, B.; Meng, X.; Zhu, C.; Liu, G.; Chen, T.; Liu, Y. $R S C A d v$. 2016, 6, 36192.

[40] Hattori, T.; Takakura, R.; Ichikawa, T.; Sawama, Y.; Monguchi, Y.; Sajiki, H. J. Org. Chem. 2016, 81, 2737.

[41] Zhou, Y.; Rao, C.; Mai, S.; Song, Q. J. Org. Chem. 2016, 81, 2027.

[42] Zhou, M.; Chen, M.; Zhou, Y.; Yang, K.; Su, J.; Du, J.; Song, Q. Org. Lett. 2015, 17, 1786.

[43] Liu, H.; Dong, C.; Zhang, Z.; Wu, P.; Jiang, X. Angew. Chem., Int. Ed. 2012, 51, 12570

[44] Liu, H.; Jiang, X. Synlett 2013, 24, 1311.

[45] Guo, R.; Zhu, C.; Sheng, Z.; Li, Y.; Yin, W.; Chu, C. Tetrahedron Lett. 2015, 56, 6223.

[46] Rao, S. N.; Mohan, D. C.; Adimurthy, S. Tetrahedron 2016, 72, 4889. 